\title{
Fuentes hemerográficas para la historia de Melilla: La Hemeroteca Municipal de Madrid
}

\section{Vicente Moga Romero}

\section{1.-La Hemeroteca: prensa e historia}

Una Hemeroteca no puede ser considerada tan sólo como "una biblioteca en que se guardan periódicos y revistas". Sin duda es mucho más que eso. Fundamentalmente, en la actualidad, se tiende a considerarla, cada vez más, un centro de estudio e investigación de la realidad cotidiana en determinadas épocas. Ello requiere la previa conciencia de conocer mínimamente la historia de la prensa y dotarse de un aparato crítico adecuado para el manejo de lo que llamamos fuentes hemerográficas y que son los periódicos y las revistas.

Nos recuerda Tuñón de Lara que "la historiạ parte de una materia prima que el historiador trabaja con sus propias herramientas. Esa materia prima suele llamarse fuentes" (1). Pues bien, la prensa debe ser considerada como una fuente más; quizá no tan privilegiada como el documento (politico, jurídico, económico, social, etc.), pero es una fuente llena de ricos matices que, quizá, serían inaccesibles, de no existir.

Tenemos que deslindar entonces dos conceptos complementarios. Por un lado, la prensa - fuente escrita, manuscrita o impresa, de periodicidad diversa, diaria, semanal, mensual, etc. - cuya importancia no ha cesado de crecer fundamentalmente desde el siglo XIX. Hay simplemente que recordar que la prensa ha sido un vehículo de difusión no sólo de la cultura, sino, en muchos casos, también, de la libertad. Recordemos algunos nombres próximos y conocidos como Vietnam, Watergate, 0 , también, el caso Dreyfus, o la excepcional trascendencia de la prensa en la penetración de ideas innovadoras, como en la España del siglo XVIII con la Ilustración, etc. Así, la prensa, desde sus origenes - podemos remontarnos a las "Relaciones" de Felipe II- ha mostrado su principal empeño en mantener el contacto con la cotidianeidad. Ello es asi porque "es el vector específico de la información y de la actualidac inmediata" (2).

(1) TUÑon DE LARA, Manuel, Por qué la Historia. Barcelona, Salvat, 1981, pág. 18.

(2) BETHERY, Annie. "Les périodiques (Capítulo $80^{\circ} \mathrm{de}$ ) Le Métier de bibliothécaire... Paris, Promodis, 1979, pág. 160. 
De otro lado, la hemeroteca como institución que custodia los fondos hemerográficos y que los dispone de manera que facilita su acceso a los estudiosos, investigadores. etc. De ahi que surja el concepto de hemeroteca como "Biblioteca especial" porque la organización y el tratamiento de sus fondos tiene muchas afinidades con los de las bibliotecas.

Aclarados estos dos conceptos, es necesario decir que la historia de las hemerotecas es más reciente aún que la de la propia prensa. En España se remonta a inicios de este siglo aunque todavia no cuenta con la atención que se merece. Muchas ciudades y pueblos, que han contado durante su historia con uno o varios periódicos y revistas, no se han preocupado de organizar centros de custodia, recuperación de fondos antiguos y acceso al posible usuario. Un caso patente lo tenemos en nuestra ciudad, donde en potencia existe la posibilidad de realizar una excelente Hemeroteca. Pensemos que Melilla ha tenido una gran variedad de periódicos y revistas en los siglos XIX y, fundamentalmente, XX (Diario de Melilla, Heraldo de Melilla, El progreso de Melilla, El Telegrama del Rif, Melilla Festiva, El Ultimo, El Comercio, El Eco de Melilla, El Muluya, Melilla Literaria, Correo de Africa, Pro-Patria, La Gaceta, El Microbio, El Defensor Mercantil, El Economista, Melilla Joven, La Publicidad del Comercio, El Liberal, Melilla, El Cronista, El Satélite, Africa Social, La Cotorra, El Comercio de Melilla, El Popular, etc.), y tan sólo se custodian dos colecciones, eso sí prácticamente completas, del periódico El Telegrama del Rif, luego Telegrama de Melilla, y una de ellas de libre acceso al público ya que se guarda en la Biblioteca Municipal de la ciudad. Pero, ¿qué ha sido de tantos otros periódicos y revistas? Unas escasas e incompletas colecciones privadas y poco más, de modo que hoy la hemeroteca más completa de Melilla — salvadas las dos colecciones citadas, se encuentra en Madrid, en su Hemeroteca Municipal, dispersos, al parecer, los fondos de la Hemeroteca de Tetuán, creada en 1939 en pleno Protectorado y que contaba una rica colección hemerográfica del Protectorado y de Melilla (3).

\section{II.-La Hemeroteca Municipal de Madrid}

La Hemeroteca Municipal de Madrid es una de las más importantes de España. No debemos confundirla con la Hemeroteca Nacional, ubicada también en Madrid, aunque en distinto edificio, ni, tampoco, con la Sección de Publicaciones Periódicas, Revistas y Series de la Biblioteca Nacional, que son las otras dos hemerotecas madrileñas.

La Hemeroteca Municipal de Madrid fue creada en el año 1918 con fondos procedentes del Archivo de la Villa y de la Biblioteca Municipal. Hoy cuenta con fondos antiguos de inestimable valor - como la Primera Relación de Lepanto de

(3) Véase: BACAICOA ARNAIZ, Dora, "Inventario provisional de la Hemeroteca del Protectorado", Tetuán: Editora Marroqui, 1953, págs. 48-52. 
1571 - y con numerosos fondós históricos de la provincia de Madrid, de las provincias españolas, de países extranjeros y de Sudamérica.

Sus fondos bibliográficos se evaluan en unos 300.000 volúmenes, correspondientes a 20.000 títulos. enriquecidos constantemente por compras y donativos (4).

La situación actual de esta Hemeroteca es muy buena. Situada recientemente en el remozado Cuartel del Conde-Duque de Olivares, ofrece un servicio inmejorable, tanto en el acceso a la documentación, como en su consulta y en la obtención de material duplicado y reprografiado. Esta nueva sede de la Hemeroteca ha posibilitado que el investigador cuenta con un buen número de comodidades y una gran agilidad en el trabajo. Así, el horario es suficientemente amplio - de 9 a 14 y de 15 a 19,30-, y la admisión es libre, con tarjeta de lector que se confecciona en el mismo edificio de la Hemeroteca.

Los fondos de la Hemeroteca están catalogados y clasificados, en un catálogo, en fichas, de títulos. Además, existen dos catálogos en forma de libro, pero el primero se refiere sólo a Madrid - publicaciones antiguas - y, el segundo, es una relación de las publicaciones que se recibieron en la hemeroteca entre 1931 y 1933, donde sólo consta, para nuestro caso, El Telegrama del Rif, El Popular de Larache y Africa de Ceuta. Este libro recoge año de fundación, matiz de la publicación, carácter y periodicidad.

Seria muy útil disponer, también, de un catálogo de materias, así como de un repertorio geográfico por ciudades.

Los servicios de reprografia y duplicación (microformas, fotografías y fotocopias) son excelentes y rápidos.

El acceso a la información parte pues de la necesidad de conocer el título de la publicación en cuestión, única forma de localizarla. Los periódicos y revistas están fichados, encabezados por el título y con datos de Lugar de publicación, Director de la publicación, Periodicidad, Carácter (Político, Literario, etc.), Imprenta, Años y Números que constan en la Hemeroteca y Signatura topográfica.

III.-Prensa de Melilla en la Hemeroteca Municipal de Madrid (5)

AFRICA SOCIAL

Revista mensual ilustrada. Melilla: (s. i.), 1917.

Signatura: F. P. Carpeta 96.

AMANECER

Organo Nacional-Sindicalista de la Falange Española de las JONS (Luego

(4) Véase: MORALES VALLESPIN, M. ${ }^{a}$ Isabel... "Nueva guia de las bibliotecas de Madrid". Madrid, ANABAD, 1979, págs. 197-199.

(5) La relación no es exhaustiva, sino indicativa. Los años que indicamos para las publicaciones son los que tienen en la Hemeroteca Municipal de Madrid. 
Semanario de Fet y de las JONS). Redacción y Administración: O'Donnell, 7. Melilla: Tipografia La Española.

Comprende: Años I y II. Números I a 58 (12-octubre-1936 a 26-121937)

Signatura: $1005 / 2$.

COMERCIO DE MELILLA, EL

Revista Quincenal. Melilla: Artes Gráficas Postal-Exprés. Director-Fundador: J. L. Ortega.

Años 1919 a 1924 (Años III a VIII).

Signatura: D/20016 (Biblioteca Nacional de Madrid).

CRONICA DE MELILLA, LA

Director-Fundador: Nicolás Pérez $M$. Cerisola.

Años 1929 ; 1936-1937.

Signatura: 656.

DIARIO DE MELILLA

Melilla: Impr. San Antón (Ofícina: Horno, 11).

Año I, número 1, 12-diciembre-1893.

Signatura: P.V.P./5.

FRATERNIDAD, LA

Periódico africanista de información y literario. Director: José M. ${ }^{a}$ Burgos. Melilla: Imp. Sor Alegría, 3.

Año 1922, número 1, 6-noviembre-1922.

Signatura: P.V.P./5.

GACETA, LA

Diario gráfico de información general. Director: Jaime Tur. Melilla: Plaza de la Iglesia (Barrio de la Reina Victoria).

Año I, número 1, 31-octubre-1912.

Signatura: P.V.P./5.

GACETA DE MELILLA, LA

Periódico africanista y de información general. Director-Fundador-Propietario: Jaime Tur. Melilla: General Marina, 10.

Años XXV a XXI, 1-junio-1936 a 15-enero-1942.

SEMANA AFRICANA, LA

Revista gráfica, literaria y deportiva.

Melilla: Imp. La Española.

Año I, Número 1, 10-septiembre-1923.

Signatura: $1041 / 3$. 
TELEGRAMA DEL RIF, EL del Rif.

Director-Fundador: Cándido Lobera Girela. Melilla: Imp. El Telegrama

Años: 1926 a 1964.

VIDA MARROQUI

Publicación semanal ilustrada. Melilla: (s. i.). Director: Fermin Requena.

Años: 1928 a 1936.

Signatura: T.V.P. 14(16); 1056/2.

Años: $1926 ; 1932$ a 1935.

Signatura: Afr. C. ${ }^{a}$ 7068-2,3; Afr. Est. 19. (6).

\section{IV.-Anexo}

En este anexo incluimos algunos textos ilustrativos de periódicos y revistas mencionados en el artículo. Permiten mostrar, de una forma gráfica, la importancia de la prensa en Melilla en determinadas épocas.

\section{1. ${ }^{\circ}$ AMANECER}

Portada del número 1 , del 12 de octubre de 1936. Número 38 del 17 de julio de 1937, págs. 10 a 13.

\section{COMERCIO DE MELILLA, EL}

Portada del número 53 (Año III), del 25 de agosto de 1919. Idem, págs. 2 y 3. Número 98, del 5 de julio de 1921, págs. 2 y 3 .

\section{CRONICA DE MELILLA, LA}

Portada del número 1794 (Año XIV) del 23 de noviembre de 1936.

\section{DIARIO DE MELILLA}

Portada -y única hoja del periódico- del número 1 del 12 de diciembre de 1893.

\section{FRATERNIDAD, LA}

Portada del número 1, del 6 de noviembre de 1922.

\section{GACETA DE MELILLA, LA}

Portada del número 3858, del 8 de junio de 1936 .

(6) Hemos incluido tambièn signaturas de la Biblioteca Nacional en aquellos casos en que ésta -bien en la Sección de Africa, signatura Afr., o bien, en la Sección de Publicaciones Periódicas, Revistas y Series, ambas de la Biblioteca Nacional de Madrid - complementan la información que tratamos de facilitar. No se han dado otras notas (Redacción, tamaño, paginación, etc.) por lo variable que han sido en el tiempo, y por la propia naturaleza de este informe que no pretende ser exhaustivo, sino, como ya se ha indicado, orientativo. 


\section{7." SEMANA AFRICANA, LA}

Portada del número 1 , del 10 de septiembre de 1928. Idem, página de publicidad.

\section{VIDA MARROQUI}

Portada del número 1 , del 10 de enero de 1926. 


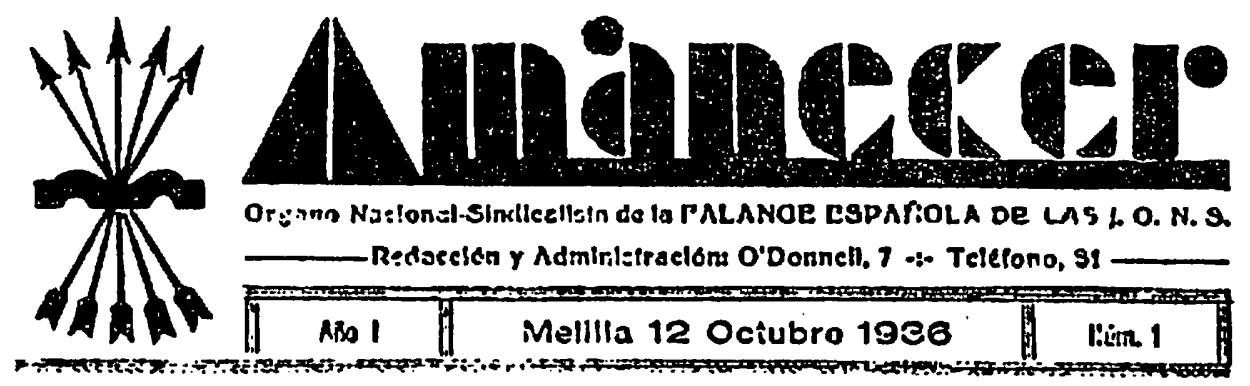

\section{EDITORIAL .}

AMSANECER... He agul el iftulo de esie periddlio, destinado a recezer fos actividades de la Pabringe Espastiola de las J. O. N. S. ea Melifts. Un ifrulo romisslleo, si se qulere. pero suave, como la briss de ese nucvo alre que erizfiezs a so plar por Espaths Darriendo tudo to viejo y eadieo....

AMANECER... Aspiro a oer en lodo momento, el porfavos de los glorias de la palange y el remanso doade repaser. despues de los penalldades de la fornads. Su creacidn era una necesidad que se delabo sentir. porque deda la misidn rrsseendentsl de Palonge. en sus actividodes soclates, ero pnvelso confsr con un drgano periodissteo, con el que de una forma lenta. pero segurs futsentas llevando hissta las mts aperfudas hogsres, fodo el confenido de nuestro programa. aun desconocldo por muchos.

AMANECER.... Ilaclendo honor a so nombre, nace para ofumbrse con lo luz de sus verdedes, is nueva ero de la vida espaciola que empezd el dla 17 de Jullo en avesire quertics Espona. No quien tener contseto alguno con ef passdo odiaso. En lo lamenso de au luminosidad azul, este IImplo y didiono AMANECER oerd el ostro. que gufe tis fosa- de Palorge Espachola eo auestra cludad poro cooperar. al resurgir de ta Patris, destrozsda $y$ hundida par al morrtsmo.

AMIANECER.. . Sishudo coithosenmente a sus comandos de Prensa y al piseblo da Mclills. enviando al mismo Hampo ed esfe selatado Dla de la Fiesta de la Ruzs, ua abrezo efualvo y condish, al Jefe Supremo de nuesira Orosinzactdn. losd Antonto Primo de Rivera. haclendo volos porque se acabe pronio easa pesadilla y en el pluzo mds breve, le veamos cendocir lo nave de Espafa por las rufes de glorta que Il solisbs $r$ que yo ven convirilendase en hermoses realldades.

LA RLOACCION

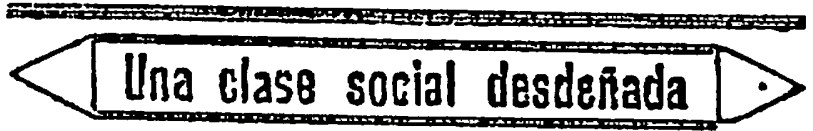

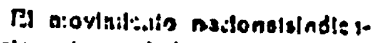

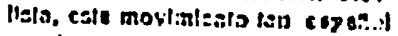
$\%$ a la vez, isn enrozeo-po.fue iebe colocorsz en ls evanzeds do

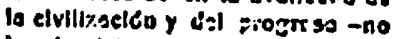

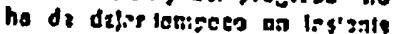

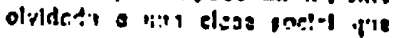

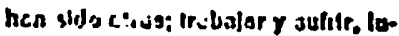
chsr y uertr tinuldes y allenneloesmanie, ruisisioin so thogar las earrecheces y la, f fsi!:-jo. Poco pora los de al ribs. i: i miroda cleraFre con e!t 2 de?:. ctlv's por parte de tins. Pars lo.s de -halo, equi. vueacannuate, fus en loda ososión una sagundy parte de arlstocracle y burzyssle. Prtslonmern entre das luesz^ enurises, uni por su dine. ro y otro por en inasa, se ha pasa. do to vido reclifiar.to if lloes o des. vios casprectos o reitrntes.

$Y$ ell eara clas: hiy. zla duds, "ה elsvido tonlo car clento de valor ve relonol; vator gind illuehrs ve. ces no brilla sobre las pler:as del irtinio popular y cuyos ineritos con relaelón a la Pairla no llegad nunea a deetacaraz. Thens merart. llosas nelividudes r."e sijgon ea lo combra; ofrece pli:a." rivd is InteIlgeaclos y rolunted es; testats. co. ire, lovento, estedta, y siscicte. slempre lieva eobre su stizs cono uo eafiguna di conldi:s'so, corso ua -ello de oproblo, lua conilecsejó secular bleo insle, que parce giltarle: -Ta, cuire y izchslo; lasora para las de amba: evitcla vase los de ubslog no lienes diresho a mase.

$Y$ en estas elrcunstancliss los de. colsdas papo el espirila de $t$ t clase metlu, es euando en el h sitronte del porventr, anumelada con esse urisente de la luche $y$ el combate de hoy: es ca estos mornentos de guerea y de muerie evondo, por prtmera ves ea la vida, euens uo clcrin de eszerenes ceren dzl corazón de esa masa olvidada: us grt to. una bassdero, una Idsa y un tropalso que heblan muy eldio y enuy fuarte de la redenelso de los medlonos, del absurzor de ons fusticla i ira los g llecutes da la pluma. 
17 DE JIIJ) UP 1436

\section{El Alzamiento Nocional en Melilla}

UIHA ACLAI?ACIÓN Y UNA IOEA

En inl ancerlor articulo (?) que lleva este misino iliulo-compucs10 apresuradameniz para dar sa. iislace!ón a amables requerinilenlos y fars ser pulticado en el antr. versario de la feclia glorlosis-se Jesllzaron, debido o la prelinura diel llempo algunos crrores, ho de muciso monla, pero st lo sulicleri:es piss que en inl deseo de siustarme en la rosible a la verdad-yia que lo llistoria debe basursz en riall. dedes-me ereo cn el diber de reclificar.

E: uno de los fúrrafos consto que los ienleniles corancles Segut y Oazapo fueron a deiener en la Restdencls al general don M.9uminel Romerales Qulnicro, Jele de la Cir. cunscripelón Oriental, afecto al Prente Popular. Ello no es e.secro. l'us a procticar esla detenclúi el Jelz del Mlovlm!ento en riellilis ton Juon Segus Almuzars acomponedo For el szalente Somanlezo. dz In. gessleras y de dos soldados da Rzgulares. Podia holer ido tainbiśn. nucstro Jele. Pero no fué. Oiro cra su comelldo. Habla que atraersa la luerza pública para el logro de la buens causd y gora eso su verbo cálido cro más que suficlente. EI puctlo de Aelilla recarderá seguromen:e sus vibrantes y aniriozos chiarlas por el micrólono de la $\mathrm{E}$. A. I. 21, plenss de sano e irreslaiible opilmismo y que tania connanza inspirarod desde loz primeros momentos.

Nucstro Jefe liobla de sulrir ln primera agreslón de la tarde en es1a Comisión. Le dispararon desde las alturas del Fuerte de San Car. los con furgo de plsiola. miemiras con algunos jefes esperaba pusedn. do por el pallo la llamads ielefóni. ca del mologrado teniente coronel Segul de haberse daleuldo al Generol.

Efectusado un reglstro en dichies casss 110 se hallo ormu de fuego alzuno, pero sl las liab!o pues naso larde y amporodos por lo obscilildod de la nochie repilleron la ngre-

slón contra las'fuerzas que guar. risclan la Comlsión.

Se lixo mucliss veces el proposlio de hutidir con borntos de mano diclias consirucelones que doinliabon cl cuariel-y se eviró una vez por hollurse en ellns una pairulla de Lo Legión que liabla suLido o reglesrurlas. No obsisnte sel se debla listuer hecho. Los pls. loleros suhion for la parte posterlo.-Parque Lobera-y cuindo se adverilan afurados se arrolabon sobre un montón da pala que ho. blan puesso de oniemano bulo las De haberse desalojado estas barra. cas y eslablecido vigilancia a llempo, no nos hubleran producldo dos dlas después una bala--no me otre. vo a decir la primera-enire la seeclón de Regulares que al mando del tentente representante setior Ocón. habla de venir en la tarde del dis 17 a relorzor las escasas fuerzas que defendlon la Comisión.

Ali. en la misma sala de dibujo dotsde estuvleron defenidos los cuardias de Asalio anterlormente. murló el Inforlunado oskarl, entre penoson estertores, solpicando con su sangre-songre derransda por Esponis-lunas corpelas de lolo. crallas ećreds de linl, como sl quislers Indicar que hasla eze pedazo de auberanla espanola liabla de llegar el ulibuto del pucblo islánilco. alzado en armas coniro la barbarie roja.

Pilos la eseasez de hombres en los primeros momentos. y otros serviclos más Imporlantes descul-

Exquisitas Gallelas velitanas de las iniseros vivlendas.

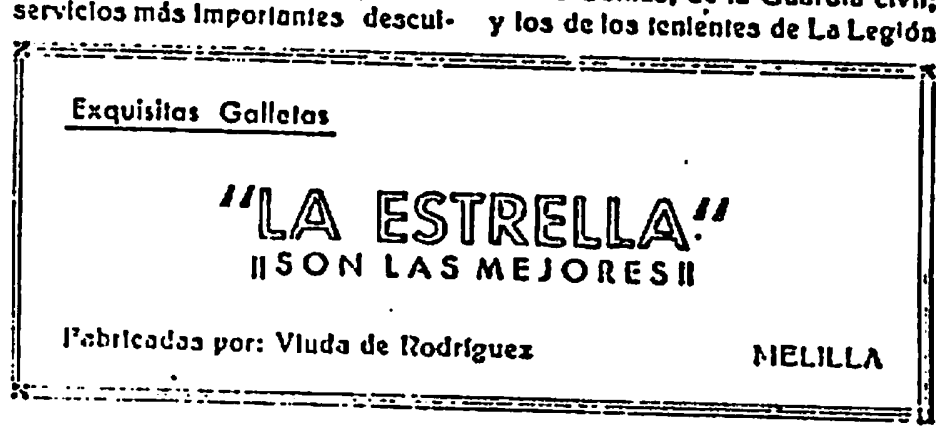

Lion esle delillz. $Y$ ounque mos istis lis smicirallodoros del Ienienle Iluzas reforon de beles dielios viviendes.. inllogrosomenle sin couser vielluns-no pudlaron Impedir esis muerte.

Los diflles dedos del tentente Lolorre-esa mano hay desgraclada. mente pzro con glorla, destrozada Dor la meiralla enemiga-coplaron casl en su Iniegridod el Bando y a redida quie se formaban las holos esiss eran reproducidas en una ralaslus en la misma representación del Tcrclo. paia liego ser leldos en la calle y lifadas por los esquinis de la población.

Tinire los leglonsilos-que como lievo dichn-ian bravamenie deiendleron el cuar!el y olros que mós turile se agregrion figurun los nomibres siguienies, odernás del sargento don Joaquin Sousn Oll. velra. quz por clerlo era de naclonallded porluguesa. Cubos: Jullo Serrura y Cristóbal Chamizo. Leglonarios: Benilo Arelnlego, José Mayo. Enrlque González, Ramiro Gullsrrez. Antonto Gurels. Jose Pérez. Antonlo Alarcón. José González. Antonlo Rivzro, José Macelra. Serolln Rodriguez, Juan illnguez y algun olro que no recucruo.

El fersonal del Equlpo Topoerd́ico se componfa, del lopdgralo senor Luque, sargento M.B. catos Garcla y Alonzo y coldados Villasana, Garcia, Bartual y Carrosco.

Eulre la pleyedz de nombres glorloso qua licrolcamente hablan de conar en el irsnscurso de la campana y que se encossruban desde las primeras instantzs presznies en la Comición de Limlies lay que enadir los del caplión Cano y 18 . nlente Comas, de la Guardia clvill: y los de los fenlénies de La Legion

"SON LAS MEJORESI

Jabricaclos por: Vluda de Rodriguex

MELJLLA 


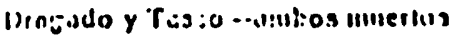
glurlisenumite cil combsie $-y$ el del ienlente Sonisnlito, de Ingeileros, que funto con sil compsna. ro SJncliez Sudrez ton pilincipol popel liablan de desempenar en el movimiento digniflcador.

No fueron muchos los folsnglslus de acclón, quz ese dla memomble acudicron a is Comision en busca de consignas y de armos. Recuerdo algunas nombres: el del - inoroo blanco era Juanlio Cirmona. Más larde lletó a inl domicillo con Cis:atracio dun Bernardo Bra. vo-qua por clerio venla cludlendo la persc:ucidin de la poltela- y lainblen esluvileron presentes los slguicnics: don Rogello Vargas... qua con Cisadraclo y olros sallecon a por provistones para la Iropa, si Irlentio ilia ngresiśn de los plsto. leros de la $\lambda$ lenzaba-y los caina. radas don l'erisonito Agulter, don Mllguel y don lost Mrilu y don Oscar Pranco.

$y$ ya creo gue nads más. Iloy en ella-despusa de lodo lo ocurrldo y de los frecisales itroleosy aun bombardzos-iodo algue en

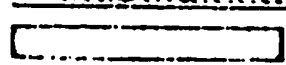

\section{Ml:zSTROS OL.OR:OSOS CAIDO3}

\section{José Sanjurjo Sacanell iPresenie!}

Uno da las fizures incs eullauies de nussilo gloiloso Eiśrclin, lo cra el genaril Senjerjo, en caitsn el fucblo ez:issol, encert:oba las virludza ilal brozo sacular éa la PJIrla, conczanrasilo en el iclos los alecios, tod.ss lis adiniraciones, 10 doj los carifos, qua el pueislo ejno. leal y comanie cle eu Palrla. senila for el Elérclio.

Su farlcla y valor eran públicesinente teconocitlos, su patriollsit o lndiscullble, su popularidad Inmenso. pucs era el findo dal puchlo. con el gue lisbli llegario a IuenillAleurse, ciminciola y slendo ainado por el misr:o, can Idolsirla.

Su error creyenilo posibla que en Espuna piaciara vivir la Repilblica bien lo fuics, con el tracaso de l.s a::ilevaclón de Agcaio, ginz le canjirits cu el paiscio nim. Ee

\section{八OOSTO}<smiles>C=C=C</smiles>

\section{$3 A B \wedge D O$}

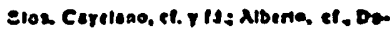

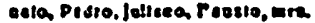

\section{-}

El Esfado podrs expropiar sin indemnización las fierras ciljol propicdad haya sido culguirids o disfruspads llegr. liminuerse.

21. Punio faleisl déla relange

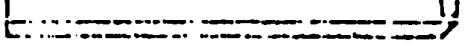
c.ilins cu li Cc:nislón de LImlies de rifiles, como al nzda hubiese sucećlilo. Mo obstanti:s las edificaclones csid́n acribilladis por la meira. lla ce la llota rola: faro aun quada Ln slllo llbre en la lachada del lilsiólico pabellón donde iuvo lugar el glorloso alrasialento noclonal. en donds pudlers ponerse uns senclllo flaca que dlicsa: « $\lambda q u l$ comenzó el clorloso reaurglr de Eopsก....

EL ALFEREZ M.B. del Disco, suirlataclo no yo cos!lgo. s!ro vilaclonics Iricomipollties con s trillilita hiliorlal nillitor.

For ezo, 110 es caircito, que cpzhas el Elírcllo eszatiol dib el grlio calividor de Viya Escano, rebzlún. icsa coiltros los eriemicos de ella, Esnitirlo qi:e alls cn su voluitarlo czeslarro porfuguć., szgufa prso a rizo lo iragchis que sa cernla sobie lispana, corrlero prestiroso a Incorfororas a sus querluaslierma.
No hables de la gueria ieon pereone alguna que no conozese $n$ l lengas en clla nisoluta confionza.

Cuando un cesconocldo to hable y te pregunte - te euente alorio hecas suceso que eea desertsdable o laloc, prlmero plensa que puede cer un es-. plo, despues un iraleor, y por lo wenos un mal cepanol. Dendnelaie a las autorldades 51 no lo haces asl, lack rrirds en crave deflito.

nosde armas, pars ocupar un pueslo de vanguardla en aquellos mo. mentos en que tania falta haclas Inteligenclas privilegladas, hor bres curildos en la luclis y corazo. nes llenos de exaltaciōn patrlólica. para ponerse al frente del buev pueblo esponol, que decidido, con resoluclón Irrevocable, se levantsba contra los enemlgos de 10 rellglón y de la Pairla.

Dlos con sus intnutables deslo. nlos. quiso que ofrendars su. vida cuando presuroso venla a entrecarls una vez más a ea Lapoía querids, muriendo confenfo por: que ya ondeaba bojo el clelo espaDol aquella bandera rojo:y iguaida que eran su fdolo y amorconstante.

Olorloso caldo en la ragna em. presa de oslvar a Espado, cola ha iarido uo recuerdo paro su themorla. en el aniveraorto de ou muerle. celebrando sciemre: farcerales por 8u almo, colocendo cororso de nores en las liffides qua den ab nomite a linrlus o calles ca diversess pobles!ones y deposltendo nores en la tumba del Insicne caldo. ellá en la tiente cluded cosiena de Esloril, clonde vivio ous difilmos dias, alento solo al momento de poder ser uitl a su Pairla $y$ donde Jescanoan sus reslos, en eszcra de elerno refoso en llarro espdfola.

miario.

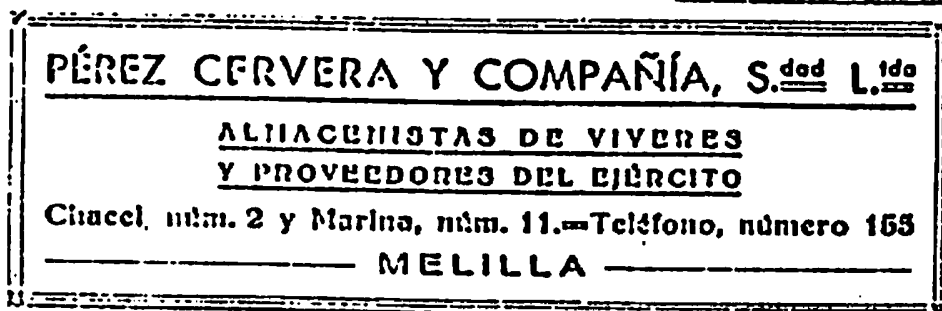




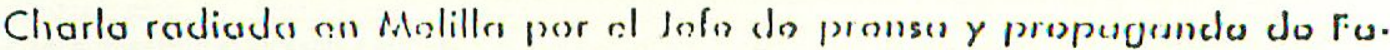 lange Española, Anlonio Montes Hoyo, el día 18 de Seplianbre de 1936}

Ni derechas nl trquicríns. Comm ron secuencie natural de ins efinismos sin inedida ocasionado por el rapitalismo. se ha venido desarraliando en lissanas en el muncón en!crii una fusta prutesta que poeas a poca ilia kunando firandes proporeinnes yue lulminalian inas ices en huelgas pareiales $y$ whras de cardeler senctal gue vellosn silviendo pura biarciolmente modiliear al:on cae cMlato de cosss. Comin siemere hass clementong al secclur para corlubar inda cuestion de somimientes hum.tnu, sicinpre cun el ca.

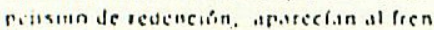
it de csols masas lus ncisiuniles que llumdndose diricentes. las encasurshon con interés unezquino de meden persunst. E:stas prolestas furmuron cl elements llamido de lequierdismn, que en prondes luclias port cossus, que en sucsenci, sun sacralisinns. fern, yue mal chemudas Y llevadas a einalsmin desmedido, sin eunidad de ningún eincroy conn espiritu de revancha perdlan su eacncis en casi laders ins casiss y datis wrins al cper/smo del capital para yue. Ilomsndose cllos cleminlos de orden puner en frente de esus antitudes una acción leg.ll gue lo revrimiese: $y$ como ni su actilud, ni su conacimiento convencis. Ia irritación de ambins quedsha en pie. isf los cosas sparece el verdadern esiado conciliacor ce rodns los derechos, que es Fulange lispañola: pero en su comienzo las derechas erejeron crróneamente que, es/a nueva organlzación era pratuliamenle su vanguardia de defensa $y$ escuda de con. lención. A tsl exircino. Ins ceniros dere. chistas ofrectan a csta infancis de ores. nización todo séncro de Jjuda cconomica: nudicnco comproterse pronlamente que nucstro propósilo no ers servir a determinado amo a intereses, sino que por el conirario, era una accín tolalita. ria y equitativis. Las izguierdas, por su marte, crelon ver anics de conncernos. lambién un proposito de cupitslismon dis. Irazede y que éramos, por lanto, nucvos enemicos que se presentaban en la palesira pors combalitlo con gallardlo: pero de un seîoritismo en el que nos co.s?undlan sin ver cu nuestra accín la es. llardís franca y leal del que solo quiere el blen para todes.

\section{La esclavifus y la llerra}

Alratarse de la modilicación del es. Inda de propicdad de ls tierts. sus por seedores han olepado is teorls de que se irata de unos derechios ian lepfilimns comin los de cualquler otra propicdsd existente. A slinple vista rarce rajona. ble esis ldea sl no se ticne en cuents que con este acaparamicalo se perludica poderossmente la ceonomfa generol Je uns nación y se sujeta a la miscris a tufint. dades de familias que de orra forma mis

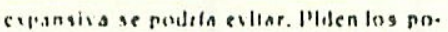
scidorcs de cirandes evienslones el re. conncimicno y pugn de su valor, bicn luese intucdiatumicnic o a pinzo determinadi: pero es el casis que nl una $\mathrm{nl}$ oira enga Jebe ser admisible porque ello ge. rla innio contno realizar un bonito nesocin je venla, con lo cual el erario que hal que procurstle la milima desenvol. luis y udo genero de lecilldad para que afronie sus oblligaciones, este hecho constituiris una carga injusta, que adeitds serviria te numento en la remora Jel necinr desensolvimienin. Decidida. mente lay que ir a la ocupación de las licrois en los grondes exiensiones que scan innroduclivas pors scguidsmente parcilada se lingun los repurtusy yaen. buniemeng que en colda caso correspunda y somecrlo inde cllo sin lardanza a uns explntacion inmediala.

llay un caso isn Inlusio, comas el de clachparminichio de las lierras yue repistra nuesirs hlagoria y que es el eanocidn con el noinbre de csclnvitud. İn aquel licmon, liss esclavos constitutan para las lamilias un elemenin inds de rlqueza que al motir los colezils de lamilis se reparilan y slgunas de ellos se le entregaba como liluela correspondiente. cual sl se trolose de ecmovientes u otrss prendas que componisn el coplial de la fanilia. Sobrevino la abolición de la es. clavilud, nadle tuvo presente el daño que ae le ocasionaba en esie sentldo a los ducinus de esos seres $y$ al quedar. Ilbreinenle esas rersonas humanas que se les redirnio de la mayor verglenza que puede exlstir en un pucblo. a la vez, se rensase en indemnización equivalense por el cómpulo que representura oquel capilal. Por lo anto no hay que filarse en esns dafios ocasionales a los propletarios de las grandes cxtensiones. por que por encima de esa cspecie de derechos esiśn los mss legillmos y sagrados que lienen lodas esas otras personas que carecen de lo inds Indispensable pa. ra suexistencia de una manera humana $y$ decenle.

Italla y Espana

Esia nación baliana ha tenido en su revolución y descnvolvimientn una lisonomba bastunte parecida a ls de nucsiro pais. Primeramente se ha reorganizado en su meirónoli $y$ ha llegado a la mbxi. ma explotación de indos las actividades. Ha ido consolidando todos sus serviclns y riquczas. lla llegado por esle inedlo a un zrado de perlecclonamlento verdaderamente envidiable. La nación lasiana posce una superficie algo inferior a la de nuestra nación capafiola. Ademśs, por lo montaninsa de su gcolngla cuenta cun bastanie menos tierra que Españs. explat.st!e y cullivable; pero en camblo. el número de habitantes es el doble al de nuegira naclón. lo con indus ins re. sorries en condiclones de uns oventura $r$ neservando de que forma podrlan pro ceder a la obligado expansión. vieron que su indispensuble ensanche lo ienisn en la colonia ce ibisinis. Todo el mua. do sabe. sl quieren aprecilarlo aln apo. sionaminio. que en la realización de ege empresa lia conseguldo dos cosas $\mathrm{Im}$ portanilsimas $y$ muy humanas. Icomo son: is de liberiar a un puetlo de la es. clavilud. Is miseria y lo ierra. como la de ismbién buscir alli en donde tonifal. unos lerrenos fersces se encisoniron sin explotsr. la necesarlo fuente de riguezo Fars con ello alender las necesidades de sus súliditos. Inda vez que en is metro. roli le va siendo Iniposible la vlda por que los inedios naturales son insuficlen. lis pera cl!n. [ista es la realidad de la a cosas visiss sin apasionamlentos.

İn cambin. en Fespaña. oliscrvando uns norm. de fusla equidsd y presisndo nos inilos a conperar ficlinente a esia olira de recongirucción y engrandectinicnto de İspaña, icndremos que serd nucsiro icritorio lo suliclente a sosicner con rclativa largueza lodas las necesida. des que el pueblo espoñol tiere. $y$ como hoy uns gran exiensión sin explotar y el numero de nuestros hobitanies es Inle. rior por lo menos en la mliad a lo de lia. lla, resultara que lo menos en un alglo scrón suficlente toda nuestrs producclón a sosicner las corgas y necesilades de la nación aun ieniendo en cuenta el pro. gresivo y nalural aumento de aus habltantes. Pni lo ianto. aventuras de expenslones exicriores o congu!gtus no se nos Impontrs por circunstancla apremiante de las necesidades de vlda.

\section{Prciczer la produce!ón acricola}

lisy uns cuestion muy importanie que constiluye uns de las principalas polan. cas de nuestrs delenss cconomics. Es. pafla es ogricola por excelencla y esta produccion no ha sldo to suficientemen. le proteglda $\mathrm{nl}$ inmentada: pues solaincnie en casos contadisimos ae ha recurrilio a trútedos cornerciales para abritle mercados a nuesira producción y solamente se ha podldo consegulr con los llamados conlincentes parciales con uns llmiliacion tan exigus como aquelles que en inclicina se llevan a cabo con cuentagatas. No liemos llegudo al naluraly compensader Intercambio de los producios en is misma parte proporclonol que le licmos Iriporiado de especles - cosis que lisllamos carecldo. alno que slenipre ha cslado en gran desnl: en la proporcionalidad ic ianto por elento Leomo pucde ser que sl de un pafs hemos adquirldo en mugulnarias atiles para las industrías cien mill!unes, eolo menle se nos lia perinlildo exportar a eae 


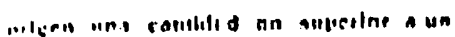

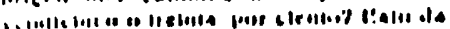
cime conecciencla que nuesiros proJuclus azrleolus no aleancen en el preciis ite sus trutus los vilnres remuneralines a que se liene dereclio. Pongsmos

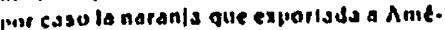
ics cn csias ennuliciones con to resirte. iuin. se ha Jado el tristic caso te mo insteser algunns anns, nl el clneventa pur clento de us gasto tolal en la explo-ucion.

l'or lodo lo expuesio es nceesarlo irie. misiblemente una ley pralecelnnista ifue -1.4igue a esas nuclones de dnnde nos : Juinios. una lusia reelprncluad, nue es ¿n une reiajria a ealso la remirla de es.1) lolircifores que en silincloncs de ."lenas cosechss un aulien dande colo. if sus projucios: y agl con esis esl. tincta ecatartua la Impusición prique is ulpd forma dejarian de hacer el netrolis.

Latlinilos y mintfundlos

Lns latilundios en ponder de unns cuanus cludsdanng que la seneralidad de - llos no ins explatan con la listensidud ive las buenas tlerras podrlan, cion como cinsecuencis la trisie sliuaclón de la t.ilia de elecurenios y la Inversión de bra. zus que to exploisise. Itstos tierras que in su msjorla gun sesceptibles de lie fos roraue con les copteciouses liechas a las rlos y formélón de ronianos se . lumenlario la producelón de una lorma mendigioss. darta como soluclon un resullado halogllsalsimo y ea, que ounen. lando el número de fainilliss emplarsulas en esos predilos. lamblén telidrismos et iruslado de esas olras lamillias camresinag que de encueriten en Isprenos cale. riles, que con la mayor nilserls $y$ todo sinero de sacpifelo. Jesurpolton una ae. cion de rabejo en superítcles en donde nunea puecien enconirar la deblua comrensución por lo inisero de lo condiclon

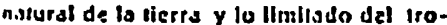
so o parcelo. En csmblo, eslos serrenos mohres. agripssas $y$ converlidons en inulinto de niayer cusmila, podrisn ser ixplntosos for los Sinilleatcs Agricolas jue los loriasen a su curco. en donde anusimenie no se hisifs mis gue una co. secha que como no conalilila el lodn de squelln fsirllils o ocrupacion, con su II. is irado producto podrls ter un increso inds qua furlese el agricutior o los Agriruttores en Sludice:o: rero nunca como serico medio de desenvolvimienio de su "rsbja y rendinienso de sus oelividailes. bip eso los latifundlos deben ser conirslidas en parcelas intenalyainenie exrlatidas y ins minilundtos nigrupsdos rara constliuli laillundios remusero. sores.

\section{l's!arec Sazuisda Linea}

lis verdadramenle maravilloso ver cómo se aj:restan al sacrinclo de forma ahnegoda is lintienss mayorts de tos hombres de buens fe de Mlelilli: y en eya segunda linea de Felange Espanola de

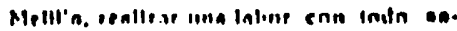

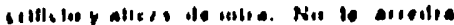
las matis nuches yue en sus cuardlis llenen que pasar. guildrubselo la Inmensa mayoila ul Jesenasu perannal y nitns con Jeirlmenio de sus Imicreses en los nelinclas. Todos eslas hoinbres que perecen velcisanss de un elitrilio lecendsla, venidos de uno cnnilnuada lucha en doncle tian obienida la prślelea de los iraloslons mbs durns. hacen inda clase de comeliclo aqui para mantener el orden $y$ ia vigilancls en indons los essos que se lea conlia. l'ero he de declr que lianio talas be observa gue hay ciesto poreen. inle de individuos que no querlendn de.

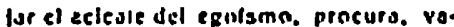
likudase de icorisa mas o menns fugilncusua, etudir ese ancrifeln. Cnn esto ac. lliud neurren los cosus que hisy que tener nuy en cuenla: La primera, que con ello rccorka de irabalo sus compone- rov que ulenen in inleme ntollgerian que

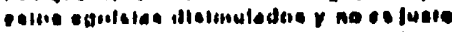
isl nroceder. parque cua ello vomblta pupone an quebruniamlento de ealud $y$ un retilamienin de violuniadea. Vean eans aenores cel relratimlento, que mnmento hilatirlca corque etrevesamos Jene en ple la Incugatie que oun no ea ha despelato del lodo; y podrle ocuprtr. nos que cual to predieaclon brblle del Ares de Not $y$ admilendo en el orden

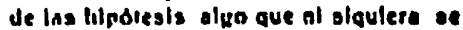
visiumbre. serfe muy triste para tocosy iamblín pare clos. el que el recurite ta puerta de esa Arca ya cerrada. tuese larde, porque el diluvio ae hable desen cadenudn. Rvilemos a Inda Irance can hiphicale de pellgro y las rezagados y egoislas se decldon, sla demora, ese allsiamlentis y ofrezcan lodo cusnio les sec porlble, Invirtiendo en ello como moneda en curso, tamblen el aseriliete

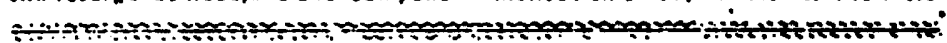
Los «Flechas》 y los «Balillas» $\diamond$

Jefatura de Mando Provlslonal de Burgos. Cin cular numero B.11 de Sepilembre de 1936.

De lormo olicial ae deterinina que nuesiras seeclones Inlanilles $y$ aus Integranies reelbisdn el nombre de - fechass. El susiantlvo ebsilliaso es extranjero. Para lo suceslvo, nuestros muchachos se llamarín F lechas, palabra evocadora arrsneade de nuesiro eseudo que slinbolles oclildad, impelu ofensivo $y$ aldn a la Palange.

Lo Junls de Mando provislonal de la Falanige Espanola lis boutlzado a lss Ieglonez Intonilles y sus inizgranics. con la polabro inecliesr elegido por au claro sanildo slinbollico.

Son los neclias porte del escudo de la Palange, y en esio lo infanclo, que visie cemiso azul, redresenta ternblén parte preclosa y ellcariso lo mús veluz y lo promesa de inna prolongada carrera.

PJeclis dice impalu, irayectorla y desilno. Y para el Impulso por el cspeclo, pars el vuelo hilrlemie de los eristales binésuldos del alre y pora el punto Misal donde los filos claven, el arquero ha de caldar au preclsión y tener serenidad de cálculo y agudeza ell los olos dirigldos al porvenir que parece remolo $y$ es Intiedlato. Arquero y arco. conlundidos, son la Palange, cducalora, ell los amores de Dlos y de in Potrla. de una gercereclón que obre mirailos nuevas a is clara luz da la Eapona renaclda.

Dellesida larea y misión sagrada son les de la ralange, inadre de los leglones Inlonilles de ilos Bechasi. Ella ho de gular milliarmeate los pasos de los ninos de hoy hacla la Patrlo de misnana; ha de elevar los pensemlentos que ohora brolan decordenados y virgenes paro Infundirles-delensoro de la noveded y pureca de los cerebros que comienzan a encenderse a la clartdod de la razśn reclén llegeda - un orten avenldo con la rlquezo de cada prlvativo truto espirliual.

Cominad Rechas. hacto vuestro desilno. Tened alegrlo en la marcha por entre el alre de nuestros ámblios purlficados por los vilicres guerrerus, los litmnos musicales y los clamores patrióllcos. Sols legi. limas promesss venfurosas ganadas fara la hora proxima por el esfuerzo desloleresado de nuestra generosa tiora actual.

Los humbres que vivimos to iranstormoción eapanola del presenie troemos en nuesiras espaldas el azole de una largo noche de Eapana cargada de mortales presaglos. Era la noche en que la Palrla lba a ser desgajada de la 


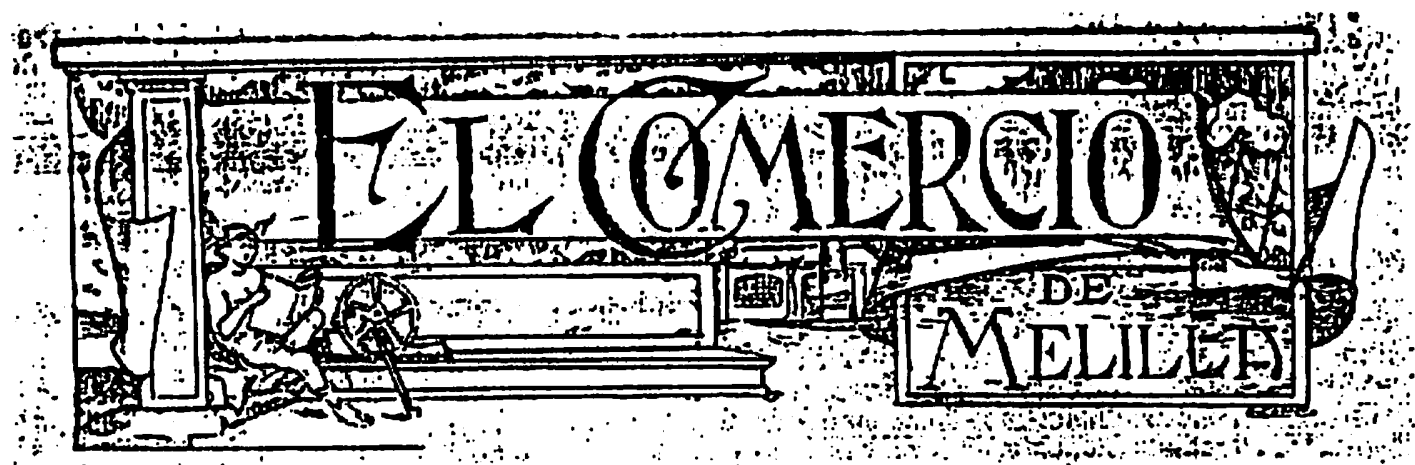

\section{REVISTA QUINOENAL}

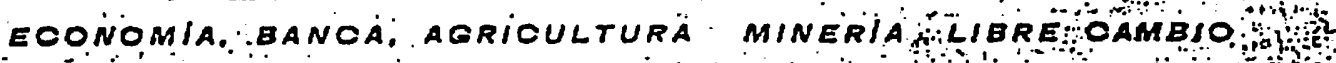
DEFENSORA DEL COMERCIO, la INDUSTRIA Y LA NAVECACIÓN Director Fundador: J. L. ORTEGa : j in:

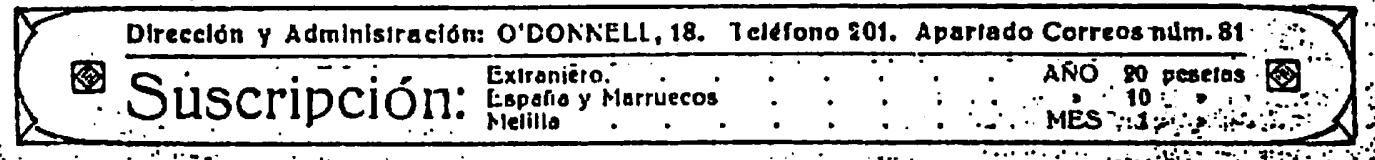
Año ll1 $\because . .$. melilla 25 de Agosto de 1919 .

1.

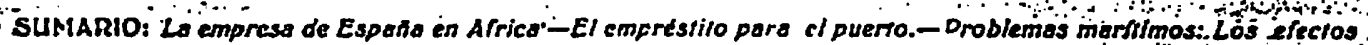

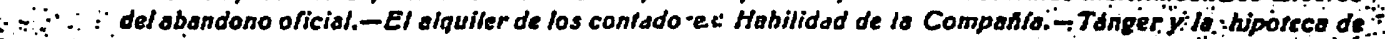
Marriecos. - Los ferrocarriles en Espada. - El comersio Hispano-Fll plio. - Nuevo prociedinicnlo para él

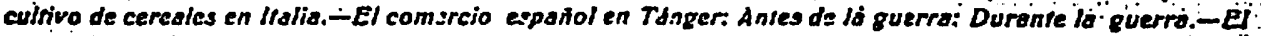
comercio con Inrlaterra. - La accidn en Marruecos, - Información lecsl. - Esfadistica del puerto de Melillä Movimienfo de buques mercanics: Movinsicnto de mercancias: Rcsumen de embarque de mineroles; - Mer:codos de Melilla. Ceura y Tetudin.

La empresa de España en Africa,

Las geturales leyes del progreso humano claman contra las falsas inlormaciones $\delta$ erroneos conceptos, por lo tanto es un deber de lodes los $\because$ ciudadanos, que sientan cariño.por su patria procurar desvanecer las allirmaciones que causan el descrédito de la naçión, y por estes mismas razón señalamos algunas de esas apreciaciones informativas que hacen en contra de la empresa de España en Alrica.

:- El corresponsal del "Times en. Tánger, Mr. Harris, acude a la insidia para desdosar la Em- presa de España en :Marruecos, si bien ahora no se atreve a negarnos capacidad y medics pa-

ra lograr la plena dominación de nuestra zona; pero aprovecha la ocasión de zaherir a nuestro pais con motivo del furibundo ataque. Jlevado a cabo por el Raisuni confra las pósiciones espa$\because$ inolas inmediatas a.la ciudad tingitana...

A riNadie isnora ya, que el golpe: de temeraria - audacia; dirigido por el. Raisuni; ha sido un gran fracaso, explotado a la: inversa por el corresFonsal inglés, y también, tris:e es. deciro; por Zalgunosi-2urdos:españoles que ;tratan de. los Fosunioside Marruecos - sin conocerle ni : por:el Jorro; porquejo occirrido no signitica.nade para

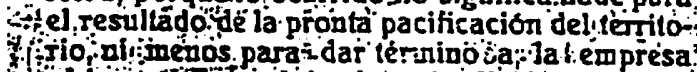

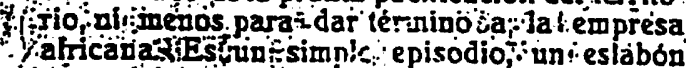

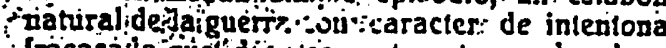
Iracasrita que d cementars' como hombres de estado yo ron carananis politicas de pequetạa monta.
Pero ya que Mf Harris se empeña-dias thasdias en sembrar nuestra acluación en' Marriueccs desde su atalaya de Tángej, buerio: sera. que pongamos su conducta en paraigón para que resalle su-parcialidad, hermana de - la -injus:-: ticia o conceplos erróneos

La impcrtante revista brilanicio Maroco, dedica gran alención en : su vultimo: zúmero a ja. obra que España desarrolla en sti protectorajo, enccmendada : al: general Bereoguer, $=$ por - los. -substanciales progresos quétobturo evi"la po-ti cificación, al vencer a los montañeses anjerinos?

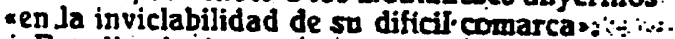

Estudia elogiosamente, : con gravíacopio dé. delalles, la marcha de la colonizacion, expre sando la inexactitud con que algunos la juzganif ya que se prescinde, en"las. comparaciones, de. factores dilercnciales: de.vivo Tnterés! En el territorio de prolección' hispánica - dice - - hay solo dos rios, el Miuluya y.el: Lucuts, de : rëgular: importancia; en la zona contigua hay cuatro; todos.caudalcsos. - La zona. Irancesa es llana en casi su lotalidad;

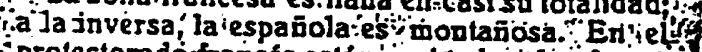
protectorado,francés están casi todas ilas famo-j sas tierras negras (tirs); je extremada lérilídad;

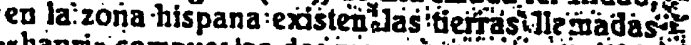

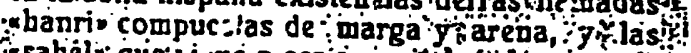

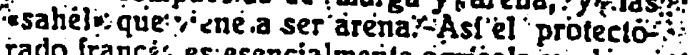
rado franrés es esencialmerite agricola y el es-

- De estas dilerencias - añade - provienen 
Potras fenciales, que dan carácter s las dos $20-i^{\circ}$

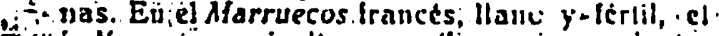
-...-indigena es agricultur y pacflico, vive-szdentario con bienestar relativo y posez medios de

- -acreditar la posesión dz la tierra. En el Ma. rruecos cspañol, casi siempre montañoso $y$ ás-. Fers, hay grandes sectorzs esteparios, dondz en

- muchas leguas no se ve la sombra de un árbol. El indigena te ella, pastor y querrero, hecho a virir en la lucha con los Sulianes a la vez q:ee del merodeo, exceprionalinente thene modn de justilicar cl dozsinio del scelo. Se ve como io fácil en una zona se hace dificilisims ell la inmedaáta. En el Allas Miznor;tan parecido al proleclorado hispanc, no le ha sido aún posible a Francia da.colonización agricola."

La revista, luego de calificar de peritisimos los infeligentes resultados que obtiene España, pone de relieve las condiciones exiepeionales en que se halla ruestro pais para la penetración económica en Marruzcos. No solo lia ganado España mucho dinero en los uiltimos años, por lo cual ëstá más furtle que nunca, a pesar de la agitación obrera. sino que puedz consignarsz el hecho importante de quz otros paiszs, al reconocer los recursos econónicos y las probabilidades españolas, reconocen también que Espaina puede desarrollarse por sl misina.

:. Marroco llama la alención sobre la brillantez con que hace poco celebró en Tánger la fiesta iel Rey Alfonjo, mas entusiasta que ofros años, y un solo la relacionn con el - nvance obienldo en la diffcil obra de pacilicación", slno que la ve unida al vehemenic deseo de poner de relicve en esle mosento parlicular, : liene España en is vida de Tanọer y la posibillJall de separar este- heche de eualyuiers decisión reicrsatk al futuro régimen tangerino.

Es muy gralo para Espeña que una.publicacivil especializada y tan inporizute, haga justi cia a si labor en Marruecos.

Es public n y notorio que Afr. Harris no jierda ripio para molesta: a Espaũa. cumo también lo es que oculta todu in que pucdis kuorecernos por razones que desconociemos.

Los conceptos de critica, cuando llevan la pasión y no son inspirados en la reatidad de los hechos, causan mayor periuicio al que los aplica que aquél a quien se pretende hacer daño.

Por otra parte, Mr. Harris- seguramzate desconoce quz la importancia adquirida en las reformas de Tánger, son realizadas desde el año 1860, en que España tuvo la guerra en Marruecos, y entonces lué cuando a la sombra de Europa empezó el fomento con el crédito comer-; cial y la preponderancia de su pabláción, : pero: siempre con el apoyo leal de todos los españoles.

1. L. Ortran.

\section{$\mathrm{El}$ empréstito

Escrito que elevó esta Cámara Oficial de Comercio, Injustria y Navegación en lecha 7 Agosto 1919 al.Excmo. Señor Presidente del Consejo de Ministros, en solicitud de que se presente un Proyecio de Ley, que garantice ur empréstito para las obras del puerto.

\section{Excmo. Señor:}

Aprobado tecnicamente por Real orden del Ministerio de Fomento fecha $7 \mathrm{del}$ pasado mes - $;$ de Julio, el Proyecto de prolongación del : Dique del Nordeste de nuestro Puerto, obra cuya ne-cesidad puso de manifiesto, no solo el importantísimo tráfico que por él se realiza, sino por la conveniencia de asegurar la estancia de los buques que tengan precisión de realizar operaciones de carga y descarga, poniéndolos al abrigo de los fortísimos temporales que periódicamente azotan esta rada, ie hace preciso que

: por la Dirección General de Obras Públicas se resuelva'en plazo breve, acerca de la lermina. ción de los Muelles de ribera, obra paralizada por la Contrafa, y cuya ejecución es necesaria -para poder dar comienzo, seguidamente, a los ifitrabajos de prolongación del dicho Dique del. T.N: E., que- según cálculos cliciales podrian comenzar antes de nueve o diez meses, si se continuara ja obra de Muelles de ribera por admiA Pero jes el caso ricmo. Señor, que cono la ifisia:

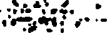

fondos suficientes para acometer, tan necesarfa obra de prolongación, cuyo Presupuesto aprobado, es de 10.813.816.34 pesetas,-elevó a la Superioridad una moción señalando la convenien: cia de emitir un empréstito de Pesetas 12.000.000 amortizable en el plazo máximo de 25 afios, y cuyos tilulos devengarian el interés de 5 por 100 anual, cuyo emprestito ha de destinarse a la construcción de las obras ea caestión y a la con-

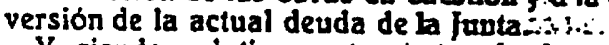

Y siendo relativamente corto el plezo que : - queda, luego de que se haga cargo de la termi- nación de los muelles de ribera.la funta de Fo-... mento, que en menos de un año estará en condiciones de comenzar las obras de prolongación del relerido Dique del Nordeste, la Cámara Oficial de Comercio, Industria $g$ Navegación de Melilla, acude respetuosamente a V. E. en

SUPLICA de que, a lin de que no sufran denora las ciladas urgentes obras y pueda en el año que resta para.que estas puedan dar comienzo, anunciarse y realizarse la subbasta, se digne V. E. facultar al Excmo. Señor Minirtro de Fomento al objelo de que presente a las Cortes un proyecto de Ley autorizando a la junta de Fomento de Melilla, paro emitir un empréstito cuyo producto se destinará a - las": obras de prolongación del Dique N. E: de nuestro puerto, en la forma solicitada oportunamente fe la superioridad por dicha: Corporación - O! i-ial,-se-:gún acuerdn de la misma lomado en : scsión de 27 de Mar's de 1918. 


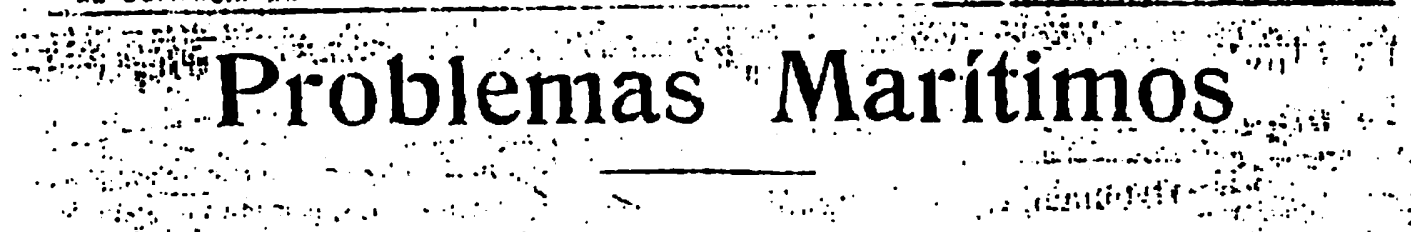

\section{Los efectus del abandono oficial}

Tantas veces hemos iratade, desde 25 tbs :0lumnas, las cuestiones maritiuns ill su aspecto. socia!. 7uo ivlver una vrz azis :?hre tema tan - lédatido y aisarseado, resulla violento para nosouivs seguramente árida su lectura para el público, deseoso siempre de la variedad en los asuntos; pera justifica nuestra * insistencia el abandono con que los Gobiernos tienen al personal de la Marina mercante, del cual para na. da se preócupan ni aún siquiera pera conocer sus males y ponerles inmediato remedio.

Aqqui, donde la imprevision hermanada con la ignorancia es fatrimonio de la politica, no puede sorprender a nadie que los Cobiernos no hayan tenjdo llempo durante cuarenta años para estudiar las aspiraciones del personal martimo exteriorizadas en inuumerables instancias, deTendidas desde las columnas de la prensa, expuestas en la propaganda societaria y a diario de manifiesto en los contínuos y lanientables conflictos entre navieros $\mathbf{y}$ iripulaciones.

El abandono oficial, no solo lesiona los sagrados intereses individuales y colectivos del proletariado del mar, intereses que en todos los paises están rigurosamente defendidos por Leyes y Reglamentos que al amparar sus derechos, les permite en el orden social, un desenvolvimicnio progresivo cuya intensidad marcha paralelamente con los progresos económicos e industriales que de dia en dia, adquiere la navegación mercante y las industrias pesqueras, sino que lesiona ademàs allos intereses racionales, atentando en el orden maritimo industria a la soberania de España.

En electo reciente está el caso ocurrido con motivo de nuestras industrias de pesca almadrabera emplazadas en el liforal del Estrecho de Gibraltar; de no haber mediado a tiempo el patriotismo y la cultura de Odón de Buen, delendiendo nuestros derechos en- aquella zona costera, aquél trozo de territorio bubiera sido internacionalizado.

-...si este ejeniplo, no fuera suficiente para justificar el punible abandono a que hacemos relerencia, otrotan elocuente como signilicativo y recientísimio, lo demostraria, al verse España excluida de la Comisión internacional que va a estudiar y reglamentar, en su aspecto biológico las riquezas pesqueras del Medilertánco. De no ser advertido el Gobierno, resultaria que una nación como la nuestra, riquisima en flora $y$ fauna marina no hubiese figurado en esa comi-

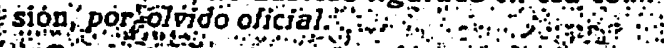
- Como siempre, a ultima hora y con apresura-

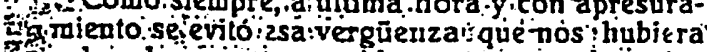
Felocado co mul merecidamente; anie! los ojos 14 de los extranjeros, sen un aivel infarior: del de 13 Marriecós, cayo Imperio no solo se jreocupa de tan importantes cuestioues. sino yue ya figura y conpera para el esludío de la Oceanogralia; penetiadn? rue del mismo dẹpende la vida de su riginara jusquera.

Dentro de pocu nos visitas al "Principe de Mónaco, uno de los más altos prestionins de la cicncia y entusiasta defensor de los asinnu: ::ritimos ¿qué juicjo se formará de nosotros cuando se le diga que en 1919 no existe aún un Re: glamento que regule el trabajo a bordo de los buques? ¿qué idea tendrá de nuestrós gobernanles cuando se le diga que las enśfianzas martmas están abandonadas por completo $I$ sus Escuelas especiales de Nautica converlidas en edministraciones contributivas, a las - cue cl Es: tado no dedica un solo cérilimo para pagar a su prolesorado; ni para adquisición de malerial de enseñanza?, qué concep to le merecerda muestros políticos cuando se le diga que no hay" Leg.atguna que proteja la vejez de auestros navegan-: tes y piscadores?, qque dira al contemplar este. pais, uno de los más ricos äe -Europá en" pescá maritima, sin un puerto industrial pesquero, sin útiles de salvamento para salvaguardar la vida del obrero del mar y sin Escuelas de pesca?, ¿qué dirá de nuestros arcáicos procedimientos para la capluración del pescaojo, y singularmente para su conservación?.

Su juicio, nos hará poco favor, peró eri parlé se atenuará cuando sepa que for falta de Leyes prolectoras sociales, por falla de enseñanza-proresional y por ineplitud de puestros gobernanles, los obreros del mar de Esṕaña emigran een miasa al extranjéro, donde su trabajo ès apreciado en todo su valor, $y$ prefiersa abapdonar su patria, antes de verse reducidcs a la miseria y. ante el vergonzoso espectáculo que se le olrece. a) conlemplar que el trálico maritimo, su inico elemento de vida y de trabajo, esfá monopolizado por e! cxtranjero que fácilarnte lo explota; supliendo la capacidad profeclora détotros paí: ses, la nulidad y el désconocimienito de los encareados de regir los destinos ae Es paña, nulidad conlesada con nobleza al preocuparse de estudiar los aranceles que han de regular la importación de productos elaborados con la materia prima, que la Naturaleza colocó equivocada. mente y por capricho de algún fenómeno cósmico, en el privilegiado suelo de nuestro país para demostrar la incapacidad e-ignorancia de los encargados de su oprovechamiento

$$
\because \cdots \quad \because \quad \because \quad \because \text { ANTONIO LÓREz.PEREA }
$$

El Comerciode Melilla se manda gratis a fas Cámar do Co imercio 7 demás organismos do cará

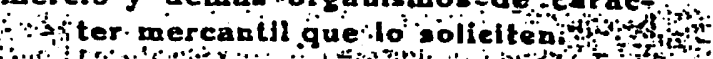
and

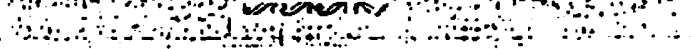


Comnili normaliuad di lus amilikes so estí restablecieyta yo ne esiudian por nuestros

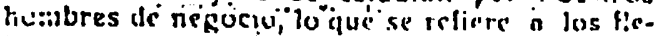
es, creemos tnuy upirtuno hablar.sobre uno te los muchos problemas internacionales: del que no puede en modo alguinn sustraerse Esp na, sus:i'ado durante la guerra y proiungadr en la post-gi -rra.

Los transportes marilimus. julı igual äué to. dos los demás de la nacion, pero este lactor de las transacciones representa unl aspeclo de la economia, eslabonados en un lodo con las evoluciones impuestas por la situación internacional, sufriendo análogos electos que el comercio y la produccion del pais.

Durante la guerra, resiringidas los comunica. ciones-marjtimás, fueron escaseando y por con siguiente elevando su cos!e.

Era entonces, unás que el albur del peligro y la inseguridad en la navegación, una temeridad lanzar los buques a cumplir con su misión.

$\therefore$ Ponjendo elevado precio a.tal lemeridad, el valor de los fleles, esto es, la oferla de iransporle, adquirió proporciones fabulosas, que gravjtaban sobre las ya extraordinarias de los pro- ductos, imposibilitando el cambio mercantil.

- Pero despues de la guerra, atravesando ruda crisis económica mundial, el precio de.los fletes, sobre la elevación consiguiente al encarecimiento dimanante del defecto de producción. contribuyendo no poco a la paralización económica existente.

- Es una de las causas, a no dudar, la pérdida de una considerable cifra de toneladas, impuesta por el aprovechamiento para marilla de gue$\mathrm{rr}^{3}$, de muclias embarcaciones mercantes; por la normalidad en la construcción de buques; por las materias primas: acero, hierro; madern: de la mano de obra, etc.; pero sobre esta causa inlluye olra, no bien advertida torlavia y que, sin embargo, amenaza gravemente la vida económica mundial: La paz guerrera.

A la guerra cruenta ha sucedido la incruenta, la solapada, la de cancilleria,yésta, sin alarde bélico, perjudica acaso tanio como. su antecedente.
Si hin Iralado por los paisrs velicedorés de con-lar " demeñar a los venciulos sin píedad " i in: :II se ha procurado el medic de cercarlos. :n tan :olo pulitica y geográlicamenti : : ycu nóminat.rente.

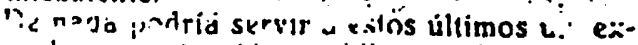
-a: o de su fiuducción, si el inles: amlio les quitaba ":jado o restringioo por las condiciones del iratado de paz.

$Y$ asi ha ocurrido que, privados de medios de comunicación maritima șuficiente con el resto del mundo, disminuido su tonelaje mercante, mermado su tráfico, no podrian aportar su oferta a las demás naciones.

Cierto es, disponiendo-de ú excedente de buques sobre los años de guerra, podrían suplir aquel defecto.

Pero a mayor abundancia de tonelaje en cada nación, y siendo universal el problema de la menor producción,-más preciso se hacia el tráfico.: con los paises centrueuropeos.

La paz giserrera, en su intento de aniquilar a los vencidos ha agudizado el problema económico extraordinariamente y el. pais debe prestar mayor atención a este asunio en ibenelicio de la riqueza nacional, ya que los bombres de pegó-: cios no pueden realizar sus operaciones sin el faclor transportes, toda vez que es el medio por el cual desarrollan sus facultades mercantiles.

Los Gobernantes tienen la obligacióni de esludiar los medios de abaratar los. transportes 2u todos los aspectos de la vida nacional, para evitar la ruina de la producción, estableciendo reglas que garantice la riqueza que representa los buques; haciendo por que el carbón se abarate en forma tal, que de rechazo encontremos la baja de los fleles, loda vez-que es misión de los directores del pais, proporcionar la armonia de intereses, que garanticen el capilal dedicado al tonelaje, dejando abandonados los buques jedicados al transporle, concediendo en cambio granoses primas a los leiroca-rilcs, eatendemos que unos y otros son necesarios a la riqueza nacional.

\section{Consolidación de la propiedad de los terrenos do labor}

En 1915 , cọn molivo de la camparia, que juzgué desacér. lada. enizrendida por el direclor de El Telegranis de. Rif a lavor de la venia de lo i lerrenos del campo exierior "de Mielilla, en la que apesar de haber ofrecido sus colum-nes pára cuario se reliriese a esclarecer el asunio. dejó de publicar, por la menos un arliculo de impugnación que le remill. jactándose de no haber tenido impugnador $y$ sacando la consecuencia de que su opinión era unónimainente acepiada y completamente desinicresada. j'a que no poseia ni un palino de terreno en ese campo. segúu

: atirmio en un arliculo publicodo en fruesas cursivas, en

Su diario correspondienle al 21 de Filireru de diclin añoy en $\because-: a$ de que no huvo periodicn lickal que acokiese ini a: :risn. publlque en . l.e l'nlrin. de Madrid lo qur. conce'pluaba un deber en hien or stalilln y sals acrirsibres.
En ese arifculo llamoho la alención de los agricultores del campo exterior de Nelillo sobre el Real Decrelo del 21 de Agoslo de 1904 al que les aconsejabo acogerse $y$ a que se constituyeran en Comunidad de Jabradores. Camara Agriesia o Sindicalo al objelo de que pudieran obtener los positivos benelicios que las leyes olorg on a ésos orga-
nistros.

Prefirieron seguir al desinierado setior que les prometía solución inmediata y que se ha hecho su Presidenie con lales proinesas $y^{\circ}$ a benelicio de la concesion de unos lerrenos que sị:io sirveri pnra cultivo. por su silución sobre In car, ztera y cerca de la Aduana Mora llegarón a tener $=-$ an valer pare edilicar almacenes $y$ ' viviendas: de modo que. carilalizadas como tierras de interior calijad por una b:icice resilltith un bonilo negocio en diano le. 


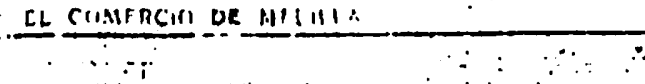

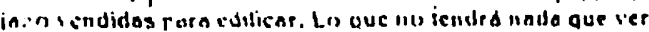
cen la Airric slyira rero positivomelile lierie mucho que ver con la esplulación de tierros.

kesullisdn de to credulidar int nunca delieron viess... los agriculiores do esle camnn exterier hall peidido laslimosamenia más de a afics : los pesclas que lian deseniboisadc pora sus mis qui inubiles y danusos gestinnes.

Si li.jt:asen sefuido mi cansxio ja tendrion iuscriplas sus propitdadss a inayor atillidomiento se hubicran alinrrado cl pogo del canun. ya que e! nencionado Real Decrelo que regulaba la colonizaciosn del cainpo exierior de kielilia les relevaba del pego y detcrininaba que a iur A sios de cumplir las condiciones que establecia se les olrerparfa escritura como usufrucluarios: esinbleciendo en. su base 8. " que el colonato es un dereclico irasmisitule por herencia o cesión o a limin onernsu, lucrativo. ¿Que inás podian apelecer?

No me to explico: comrs un lie poditio explicarme cumis ese Real Decreto que iuzaus resulvia la consolidociún de la nenviedad de los reerenns de lebnr. en cuya opinión me alirmn por rubuslecerio lis comneiente y honorable del muy! quer!do notario D. Roberno Callo, ni se ho cumplimenlodo, ni las interesodos en nblencr sus bene?'cios a ci se hall acogldo.

Ácaso las 1." y 2." de sus bases expliqueil tan raro fenomeno presumienda cuanlo puede la codicia: vor la 1." se ordena que ae divida el campo en parcelas cujas dimenslones no exedan de las necesarias nara el monienimiento de una lamilia, y por la 2." que el colono no salislará cencu alguno.

Si consideramos que desgraciadamente los ogriculıores humildes los que se conleniarion coll poder subvenir al sostenimienio de su launilia son encogidos "de carácler y escasos de instrucción. no nos sorpsenderá que no se hoyori movido. y sl, por oira parte consideramos que los consecionarios inteligentes y de buena posición no puede alagarles el que solo se les otorge el terreno suficiente pora el manjenimienlo de una lamilia, por habcrse hechado orras cuenlas, lampoco puede sorprendernos su quiejud: Si jenemos en cuensa que por el cumplimienlo de la bose 2." la Junia de Arbilrios y el ramo de guerra perdian la paric que les corresuonde del canon, lampoco puede sorprendernos que no hayan ienido inlerés por su cumplimienlo ; el R. D. haya resulrado letra inverio.

De todos mudos lo cierso es que es deplorahilisimo que no se liaya cumplido. porque sobre el benellcio que hubiera resuliado a los usufruciarios, que ya tendrian consolidada su propiedad, es que al cuniplimentarse hubieron podido pedir parcela cada uno de los horielanos de los huerlos de los cuepros y orras persorras colectivas. que son ins verdaderos agriculiores de esie campo. los que con su sudores y hasid eon su songre han hecho laborables los eriales y pedregales que rodeoban a la plaza y cuya sliuación no puede ser más precaria, hasia el punlo de que, al presente, el que podemos concepluar como el veterano de ellos se encuenira despedido, en lnema que nos resistimos a creer, a los $\mathbf{3 1}$ años de ve::ir cumpliendo religiosamenie sus obligaciones.

Con janta gestian, Innta palahteria y lanto autohombo nada ha hecho por eslas verdaderos siervos el namanle redentor de lös agricullores.

$Y$ ro se digo que con el proyeclo de ley iresentado al Senado por el Sr. Mlinisiso da llaciendo queda resuelio el problema de la consolidación de !r propiedad ell Melilla prique, si llegase o ser iry-que no lo serj-. serín bencficioso a los que vulgarmenlt se dice gue lienen el

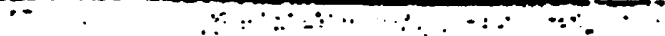
tịndin lien cubierlo y por lo sanio podran coplsalisor unnicdialamenie. Dero paro los pequefios prozletarios.para izos serla in $u / n$ erueis al inal del cual resullardn cruciliçndos. Y' en cuanio a los , no legales. pero si saciosanlos derechos-ri !ns mencionsdes hosielenus. nodo, absolulamenie nado se resivelve.

$Y$ esn hay que resolverlo e! cueda algunn intuic.on de iusuicia en las allas y balas eslerm- .. $\because$, ; .

- Su unica soiución rs n!: :i Ésladn. viurgue a lo funta de A-bịrios. recresenención aclinsi del puebla de Melillay

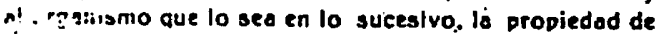
lins terrenos comprendidos en sus linniles. que el presente onsee: que esa representación-los cediera a su vez con arreglo n los condiciones que eslablezes inspirandose en el crilerio de los preferencias que la equidad. La justicio y la conveniencia recloman.

Y consie que esa donación seria compensocion. pues anarie de lo muclio que gassado la lunia de Aibitrios en Ios pabclianes del Reloi. Fuerle de Mario Cristine. Cuarlel de la Guardia Civil, pabellones del Buen Acuerdo. lus de la manzana en que eslá énclavada ta Comandancia General y la limitada por las calles de Prim.: Ceslilleins, $O^{\prime}$ Donnell y Arluro Reyes, to ha hecho en olras olorns de imenor importancia cedidas al Eslado. Compensoción que puede ser generosa. pero compensacion al tin. como el Esindo lo tiene reconocido ol ororgar a la Junis de Arbilrios por los gaslos hechos en urbanización el $20^{\circ}$ por. $1(10$ del cánon y de sns capilalizaciones.

Eslo bajo el punio de visla materiol y pór lo lanio conlahle. ¿Y bajo el moral. que nó debe el Estado a esla ciudad en cujo escudo o escrijo lo divisa de Aiuy Veliente $y$ Muy llunanitaria?

: Peru es que Nelilla se aheea deniro de sus esịrechos. limites y asl no puede realizar armonicamente su eiemplor destino. Para darle esa posibilidad precisa que se cumpla esiriciomenie la clóusula del iratado de. paz por la que se delerminaron sus límiłes, eslableciendó que para siu se-. guridad. esto es: pera ponerla a cubierto de posibles conlingencias can los fronterizos, sus limiles lós señolaria el alcance de un cafión de sitio que disparese desde el

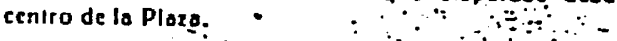

Como esa es la condicjón y el alcancé del cańón ha aunicnlado considerablemente, es necescirio hacer lo debida reclificación para llevarlos a donde alcance un cañón de sitio a fin de que se cumplo la finalidad de lo estipulado. a.:. .... . . .

A los terralenientes que queden dentro de ese alcance y prefieran naluralizarse espafioles. déieseles iranquilamenle en sus tierras: a los demás, que el Sulión les indemnice. A lo que bien obligado esib, terilendo en cuenta : que Dor sosiener su trono y su auloridad ha corrido ianfa sangre espaniola y lleva sorisfecho el coniribuyenle espafiol mis de 1.500 millones de peseiss.: que de seguro no los valen esas aridas rierras. $\because \ldots \ldots$.

$\therefore$ La ocasión es propicio: Se esid en pour parlers acerca de nuesiros derechos sobre-Tanger-sobre-los que no debemos hacernos ilusiones, pues, teniendo ya España la plaza luerle de Céula sobre el Estrecho de Gibraliar, no han de consentir las naciones inieresados el oforgarnos olra. al orro exiremo de dicho Esirecho. Por jo Ianio. delémonos de patriolerios, que pueden ser lunesias, y reelamicmos lo que por razón de lo eslipulado hay. derecho a rech intar y es de alia conveniencia por afectar a la" seguridad de Melilla y iener camo resuliancia la posibilidad de colonización. hny perfec!a sente ilunoria.

J. G. VIOAS. 


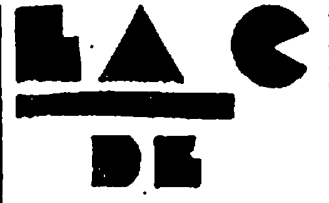

Direclor, lundador y propieserio, Nicolás Pérez M. Cerisolo

Hacla la nueva Españ

\section{Las valadpas del Genepal|lsimo Franco}

Con un recio pri isiplu rspaisoi, te francen v leall r.ije tradicio. nal, inspiramilove en un houto sin'ir de nus:ar. Ilisisila. cl Ge.

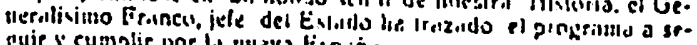
guvir y cumplir vor la nurva Fisp.iñ.

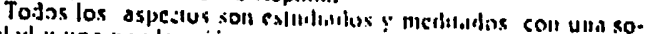

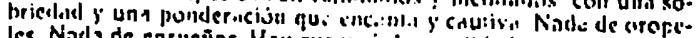
les. Natio de ensiseños. Hay que vivir bas reniidiules.pe ro unas rea.

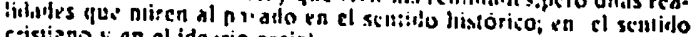
coistiano $x$ en el inentio racial

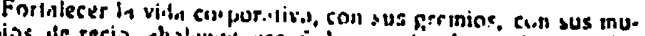

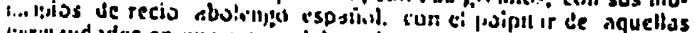

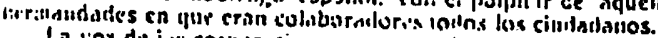

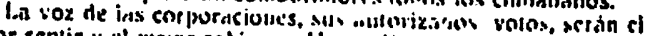

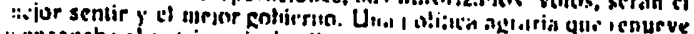

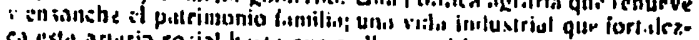

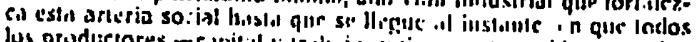

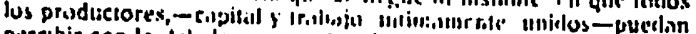

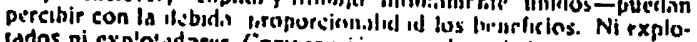
iatos ni cxp!oiadores. Conipren,ien soclal y crislianli que asocie

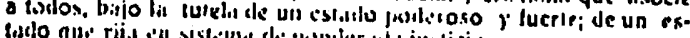

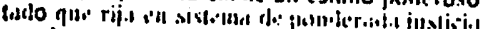

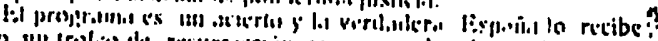

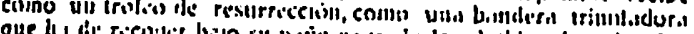

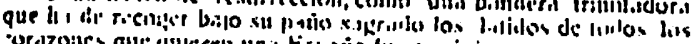

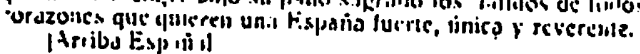

\section{EL ESPIRITU RELICIOSO MELILLENSE}

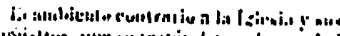

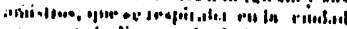

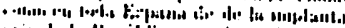

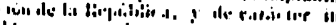

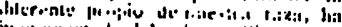

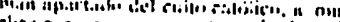

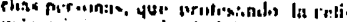

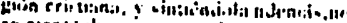

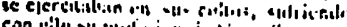

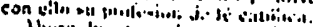

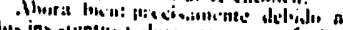

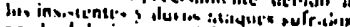

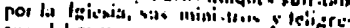

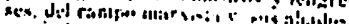

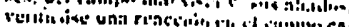
lǘi,u, yue inicios al l.

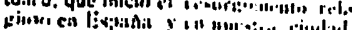

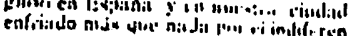

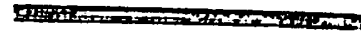
Arol

Nejer, entress tus alliojas pa-

ra el tesoro de ha Patria.

Espraisa lo inerece todo.

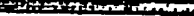

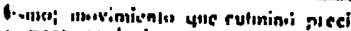
t.rmenzu en la irroen en spue con nusia

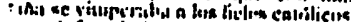

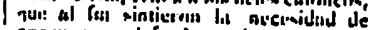

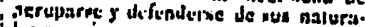
ives ensmigos.

if comm cunsecuenein laptica, de et. len hechos, ins calshieuss matillenxes of unierna con cn caldiens motillenxes of

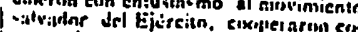
-ialvillar del Ejércitn, eixipletaring eor liwe aus herinanua de Ciacarra, al triniblo

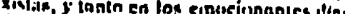

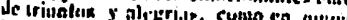

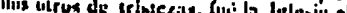
relugia dection el der, pars dor esencins a bios por los h. M.-18,419

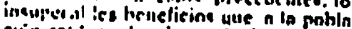

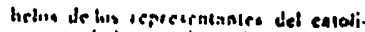

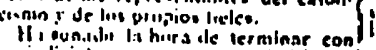
periudicialer npiutamentos. indhteren

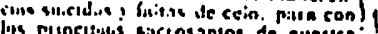
lis pitneifibs anctoranios de nuestes. relisuin y eu culin, buarutrJn con celo y critusiasnio en la lair cis. Ins benelicios ue cals dizprensa a bue liples hijas. Melilla icen-nde a au lisiorlal enso. lica! Iodoe on cituctal fucibirsin los benticius que a len puehtos repierla el mitntenumicintn de ins eacensanius pripcipiab yue hierejaron de sus marores. M.เиı.tso U. Ak.coxf.s

\section{Mellilense:}

Acude, como debes, con to impreseindible ofrends para el Aguinaldo del Soldado.

D.l moneste

\section{Marruecos vibra con}

\section{España}

Cirleren: nvisne:in los dias. Inda la

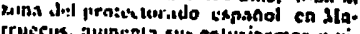
rrueces, numenta suv enluainsmos $r$ viIr" coon ous lealce enraynnes of ritmo de la nues, Sepias,o al ritate del clo.

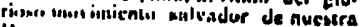
1.111..1.

l.

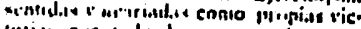

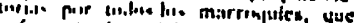

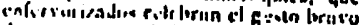

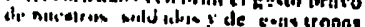
re $y$ arnis rn ci connibulte.

Mapeu:en. allwita y quiere a tispnoda

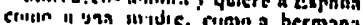

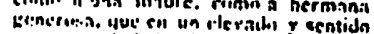

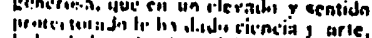

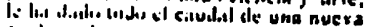
eivilieneivinis.

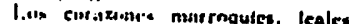
siemive. no mivilun nutetea ofrenila

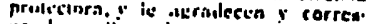

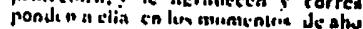

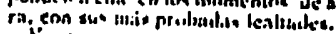

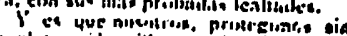

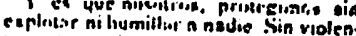
tas cullura ni miallesilar ecligioner. Pro

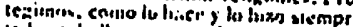
lojo calinalicro expanal, Itendienda ta mino en gestn generosoy lesietero $\rightarrow=40$

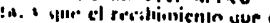

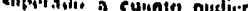

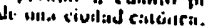

inistarnmo n exene precedenteo. to ruit entui sat ha de promlucir, in eatun.

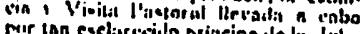
mir tan eeclorrvido prineipe de la lygle. iiz. ir Aletitla suptrarti ennin en indo. in il ordea religioso, los mayares

\section{Notas del momento}

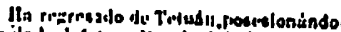
ie ile la defalura l'ruvitiel il do Palatino

as.

El notalsh ortisus don I sila Jurrdo $\dot{r}$. reja ho crazsilo rea eclehrado ariorno up reirato-execlenie prueha do dlbujodel que lue sloriow o liprideo General den Jose Kanjurjo (q. ... p. d.)

Le cluda olve el mur clozlads por todos.

Pata convalmerce de iu berida ha lie. Rinlo ol lilxarro y Gravo Teaieute Coro. del do Megulares, IHIzzlad. ceros.

lor la aolathle naruparion artiolica Pro-tijdrelie gue rize ol poren r ootablo

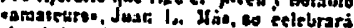
el podzicoo dla $Y$ infilinte function teatral ea ed lealre Liranal.

Fil próxime dis 1 eo reubir: is Junta do l'rutorrido da Sleaporen, lixjo la presl.

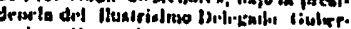

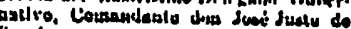
Santiaino.

Ila mejocade da la serarn deloweia quo

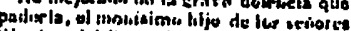

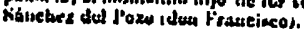

I16 Ilrzado drl trewle, el eullo medico

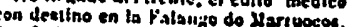
doil hule Koxes toumery.

rio encuettre rado:rma la diatiuguids ectora da doa Juati iketwalat.

Sis eolelurarou solemined funcralies poe

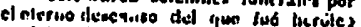

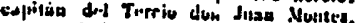

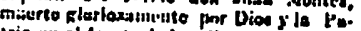
tria ete el freale de lastalla.

Al juadoso setu enicursieron las aute ildadoy y ounceposos connpaferos y ami. ces del que fue bearo oblicial, quo teati.

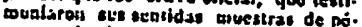
come a tos bermasica det lituado de po.

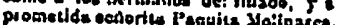

\section{Rafael Martínez Casas Matorlale de Construcelon}

Cemento Landiort

Alvaro do Bazsn. nam. Is - Tolotonoinam. zeo
Ila side somilorado telo dol territorio del itlf, al cealenta coroed dua Hegual
Pueje.

l'ars of azeole do Pollele del Protecte. rado don Joos Qulanere, ha sldo pedids Is mano de la eneantedora celiotlea to ria lutanica Xives, hJe dal comared asto doa Pedro folanie.

II. paeado deatinado al Gohneto do Prones y Conurs do ests Cireanecto do

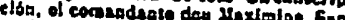
tos Uiertia. 


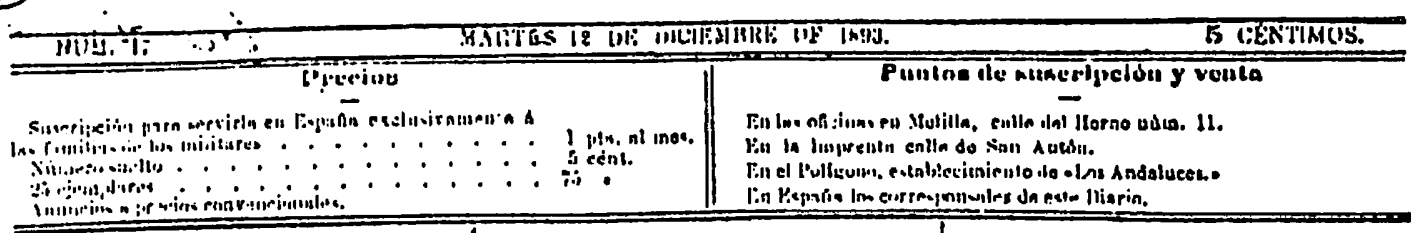

\section{SECCION OFICIAL}

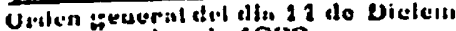
larede 1 Sese

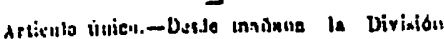

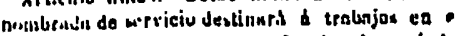

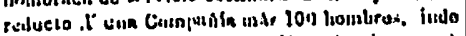

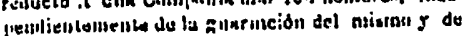

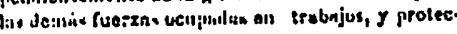

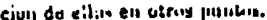

Lo que do uidell do s. li. se bace sibur un la goo

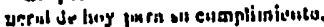

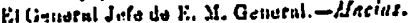

Serevialu poin of din 13 de mulerinboc de IsU3

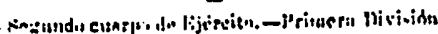

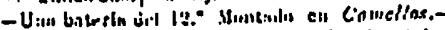

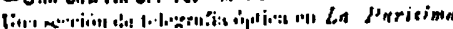

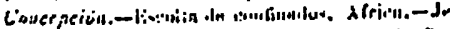

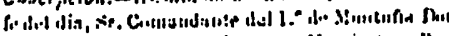

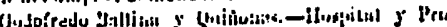

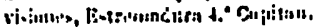

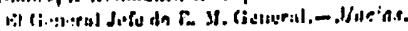

\section{ESPAIA-MELIELA}

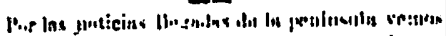

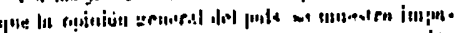

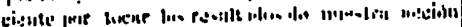

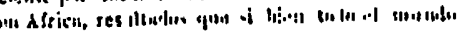

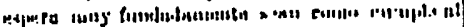

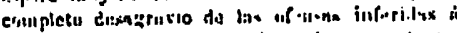

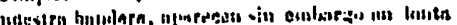

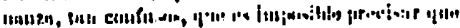

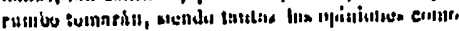

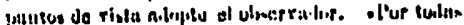

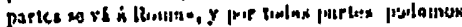

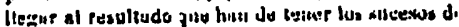
Slelilla.

Poro, alio:o bien; xul enmo en libatsu glua patible.

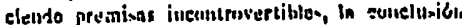

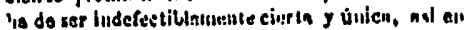

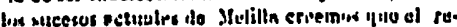

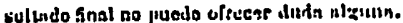

Cunndu en Stelilin we ens:urutrun unis do 2n,000

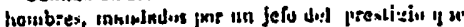

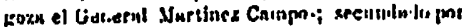

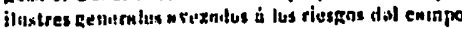

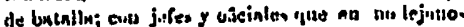

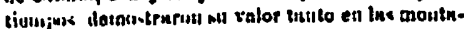

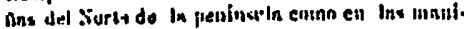

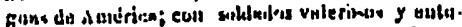
sinsene por lis iden du In patrin; coll tuatorial dr

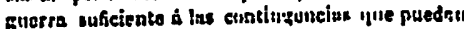
prewiltapxpi $z$ on las agues do stotilla buques do eniabata quo ya lasa dudo galtordas tnuesteus de gus los dirigen diznox sucesores del iumurtal Jour

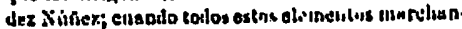
do al alulono convergen ea un pistifin, sillal es th

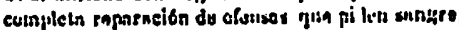

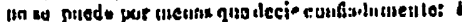

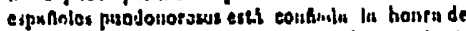
is psyiry ellos sobedn muntenetla c:ounpro ined

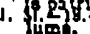

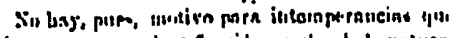

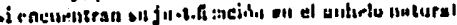

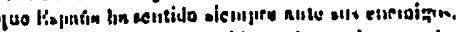
or insuficienle pinrs quo oblizan $h$ inureliar pasp de

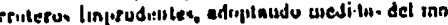

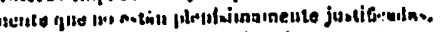

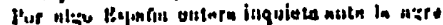

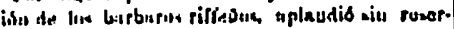

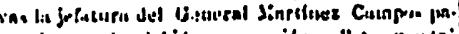

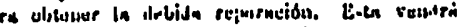

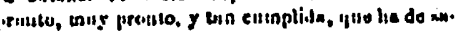

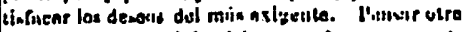

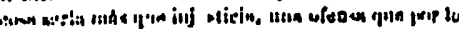

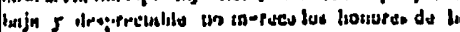
rofutacion.

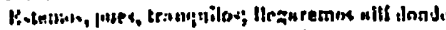

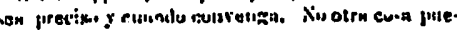

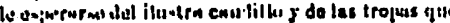

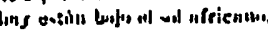

\section{NOTICIAS}

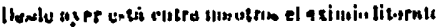

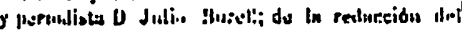

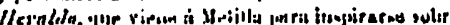

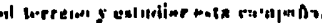

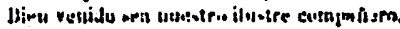

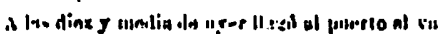

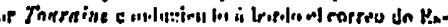

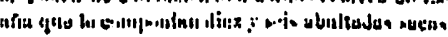

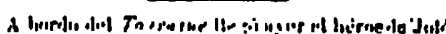

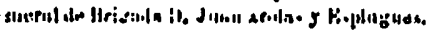

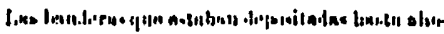

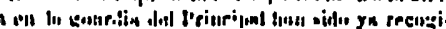

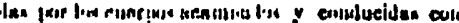

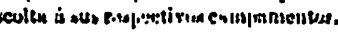

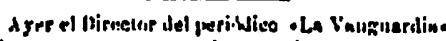

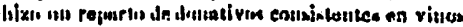

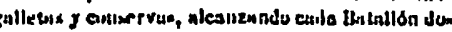

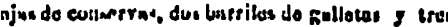
barricess do vilto.

lle xilo uniglingunente censurah una enrice-

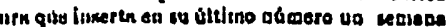
io fastivo inndeflecio.

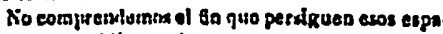
roles quio publienan tnlos cosas.

Provechn, quilete to dtn.

Lo que no puess asezurar as gqu no to dip gi honpa ui gluria.

Entro las peridilicos que llagaron ajor oo ol ecoren que cuudujo el Touraiac, liaj uno setenido a in Aduninistrucion, en euja fuja dice:

Sonor lun

Bisley Jurofa

Lo redarción inblo mandar halpudoo que lo the aro a Frajars juara outrougarlo an propla meno.

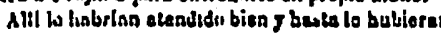
grolado una sauts.
Lo llo zalla in aupstom piserto ayer del efueero

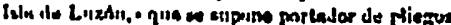

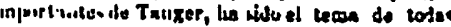
an conving sacisusen.

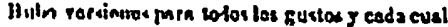

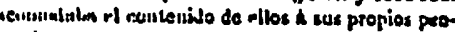
miniesilus y dimeer.

Ion unrsibis i quen oo dulin mayur céditu ora quo

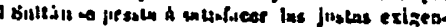

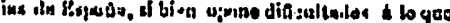

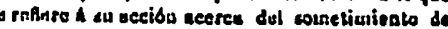
as kinblims.

Hsto sin erabarao no es giszana doveded. Hece

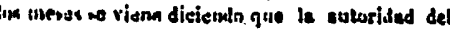
Sulluth rs vineutillo en of nifl eu todas aquellat

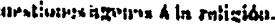

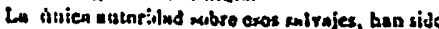

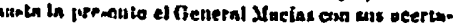

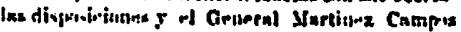

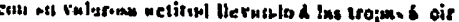

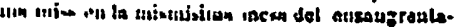
20.1 iriuch.

Aurels: wurpis el rume de quo Juley Jarefo

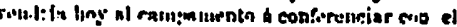

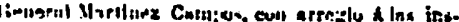

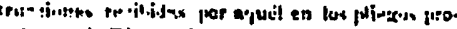

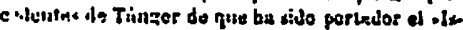
In 1.. Iotrioti.0

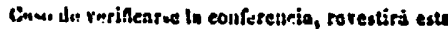

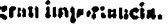

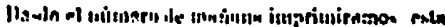

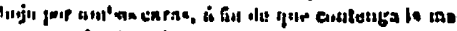

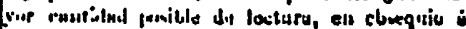

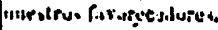

\section{SECCION FESTIVA}

Hels el cainfutiniruto:

- Oro, cliquiv, itú enbse eso do la ordenanza, und en?

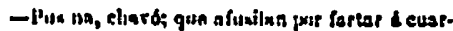
guier cosw......tueguv al matseho.

$$
\text { HOVIAIEHTO DEL PUERTO }
$$

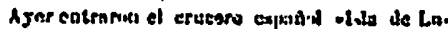
xisio priverlento de Tinzos, el rapise expmfiol di la

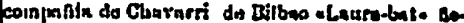

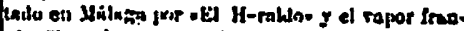
cót - Tnuraineo qua madajo el correo deudo elswis-

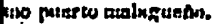

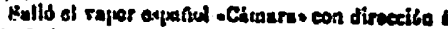
Chafarinea

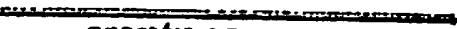

$$
\text { SRCCION OB ANUNCIOS }
$$

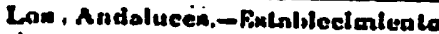
o risos y consertas Procios móstions.

Bepectatidad en Valdepetlas y colerasda Kontitle.

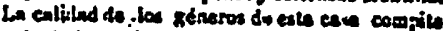
ros la de les inejores do stetitha.

Bu al Pallsobo Junto al casico do Jeles y Oscio. es.

Iraprentu del bianto ue Blatu. Callo sus dalea, dietilta. 

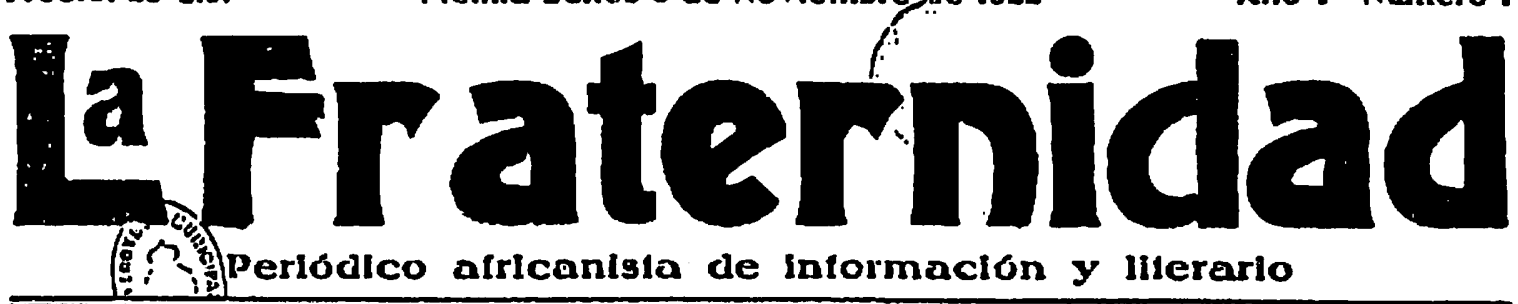

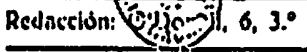

africanisla de intormación

\section{lilerarlo}

\section{JUSTICIA IGUAL}

\section{para TODOS}

La nursa ds que el Siprema lle Guc. rra $y$ Horing lis slecitislo procestar a

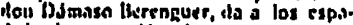

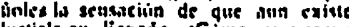

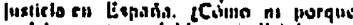

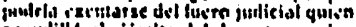

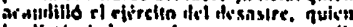

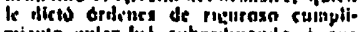
micito, gulen lut subnritumasalo a cus propins glowes la obrs irifitse del terie

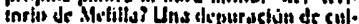

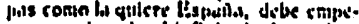

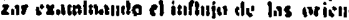

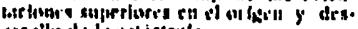

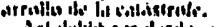

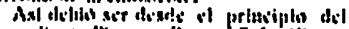

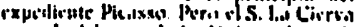

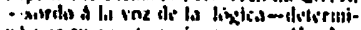

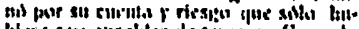

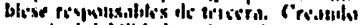

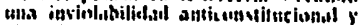

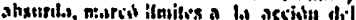

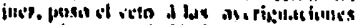

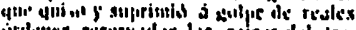

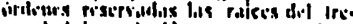

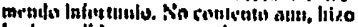

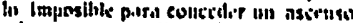

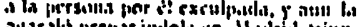

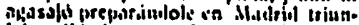
lat recibimiente, esplicudir,", apoficosis, comio sl to ilsestruction de un egirtito putcilera scr cosa ojeild d sa woneral en prle. Entralanto, pur decisivies del setior Ls Clerva, lbase comenzando a suma risr d los subordinados inl esudilts.

La monsiruosi teorin del cacinjic murciann epa de imposibla sastenituiels10. ¿Cusado se vio cn al mindo anums. lia lun inverosimilcomo la tmpursts al se. nor Picasso? Los teals's leciretos nomibrutlore's ts gelicral est |cle " alto comisatindesigmban explle itaincute acsic por respunsabic de cusato acnelstices en nurstro Prostetnrado. ya rll to militar, bien en lo pelticico. Ei Rephinetrento de cosponsabifidad oropia ile un seneral in lete. Las Ordenanzas millinres se muesiran astmismo explietias... Espania ie debe al gerieral Aguilera y sils muriifsimos colecas del Supremo el triunlo de li legalided: en el ejereito van aumentando has responsabilldades a compds que sube la lerarquls.

Y no vale dacir en pro del Sr. Lo Clerve, que se irs'abe di un slmple procelliniento gubernativo. Si cl Codigo de justicia Aliluar delermins que jalo cabe valerse de los proceditinlenios gubernslivos respecin a lintas que no lengyn Inlole de delilos sin suncistil th ha leJus, ccotno enes|ar en aquel urocectimienilo lodo in oruerido en lo Comantanela gevieral de Aiclilla? $Y$ conito expliearse tampotin que sed un Ministro quien bastarder las atcrinunelones lu. Jiciales y decida tn yut punto romienzs o coneluye la respansabilitlade Pareso Ienla que vetiose a titrrs la obrs indiscelpable del polileo murciann; por eso tenis que Inquirir ta juslicis si vil desas. Ire sobrevino o no por ctipas del man. do supremo

¿Qus? iAedso podia dcherse sin cab.al exelurecimlento lo orurridn destle is loma y uerdita de Mlonle Aburestn has. In la rendicion de Minate Arruil lla de averliguapse porqut it -considersbant
Director: Jose M.* Burgos

Imprenta: Sor Alegria, 3

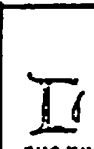

\section{NOSOTROS}

ECTOR: ociosos son los prograinas y expresivas las obras; ni queremos importunarte con aquellos ni aspirs. nios a ints que nos juzgues pop nuesiras obras. Mlss que nunca, hoy lo preciso son lieclios y no palabras. Por eso no hallarits nipul propramos al uso.

Li) nombre de este nuevo periddlico que viene a servirte, alsorra loda labor explicativa. La Frafeasipad se llama, a la Iralernidarl de cuanlos viven en Alelilla ha de atender y en nom. bre de la traternidad habrat de opunerse a cuanto la perturbe, sin que por ello srea pecar de paradojismo.

Quien nos lea hallord ludependencia, honradez, altruismo y cullos stimecro a la verdad. Sabemos cuanto vale esto y lo mucho n quic olblign, de ahi que to escribomos al Irenle de nuestra obra como la mefor riccutoris, contiando en que pronto lo confirme la แกvinivit pủblica.

L.iterallurs, politica, infornaciones varios, critica de cuanio lo murezes: lie phi to que comeuzamos a brindarte, lector amigo, seduros de que perdonarás los delectus que hoy adviertos ell muestras pejginas y olsecicindote poner los cinco sentielos en la lobor parn tuie vaja a mejoris y no te arrepientos de lounenlirla. Queremos que la Fraskanibad sea digma de if, de Melilla y del homrado lervor ds quieuss aportan su halenio a in empresa.

Dicho esto, mada nus nos queda por ariadir porque de sabihlu se calla que la fraternidad no reza con la deficiente gestion de los asunios publicos, con las demasios de los necocios par. ticulares ni con el i: jso continuo del neinbre de la ciudad para cosas que suelen beneficiarla muy poco, moral y materialmenie. ros locsles.

sulicieales - las tropas de Mielills, tan10 mazzoquliss somo petuinuulares, cusando Silvestre solleitiba reluerzos; to ocurtido i boreln de un buque de gupers ent la Iomasi entrevisla Irente a Sidi Deis; los sizoars por que se impuso inmovilidad suisilla at las luarens esponiolas despues de las coilibales de principio de junlo: las mullyos que impiclieron envlarle

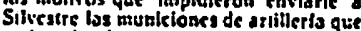
rielamiabs itesde antes del encuralro de rislamisbs lessde antes del encuealro de que los socorros pedidos no llegasen a Mitelitla hasta luego de evacusarse An nual: las razones que impusieron suspender la retlesida de Novasro; tos mo lives le no llevarle socarro a Nador, ni siquiera intentarlo, de no socerser da ze lusn. de no socorrer 1 Mlonie Arret

Para la avertiguarion de todo eso y de otras cosas relacionados con da catsstrole, iqui cantrino queda en donde no se crieuenite al general en lefe? Por tan to es seguro, indubituble. que el Senado coneedal cli sugticstorio. No ha de cons. eederial Ifin tin aqui se denepasron peticloires de anplicatortos relotivas a deli. los de oplnlón in:pulables a sensdores un liny ciernplo de gue la alto Camars - ni olrs nimpuns del musulo-ezieneito se la invinlabiitdad del carco de seno dor $\$$ hechos proleslonsles ajenos en ab bointo a las cuestion:s porismeniarias. $Y$ his de concederlo, ousms s. porque la primera pirsona d quien ello conviene es sl general Derenguer. Pues dicen sus amilos que es extraño en absoluto n lo ocur rido, ningein campo miss o proistilo vara lu derriosiraclón aue el sestno pslista ue de la justicis, donde sathe aportise lodos los cloevmerins ofista!lis, lociss las pruebas catecanteas ne-

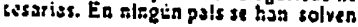

Iado cosss acien la tribuna parlamerr. laria ni en ell llwro. Pof triste. por dolo roso qile sed. no hay oiro medio que el de la acción juólciol. lo mismo para el saudjillo y pors el tultimo ollkrrt.

Nodie perisard que exlste sevicia en In Iramilacisty del asunio to cusl cons. titcye solida garantla pora el indlciado de culps por 21 Supremo tribunal castrense. El sprior leremerer se ha delendido en is Alts Cdmars, sin IImitarienes de tieinpo ni de pruebas, oun antes de que un juez le presenlara sl pliego de precuntas. El pencrol Derenguer ha pousdo lomarse alyuiros meses pera responder a las que le dirlola el fucs ins. iruetor y hassa servirse de letrados para su riplica... El censeral Berenguer hara sabido Ineluso revolverse en if Senado I in los peridedicos contra ef Supremo de Guerra y Marina. El peneral Gerea. guer no ha necesltodo slquiera suscribir el libro que se onuncis estaba escribirn. do, pues otra persona dijo lodo to que tl dirlo y publices los papeles que di hu. biers pubititisto. $t C_{n}$ be miss extensa plitud de acrion detensiva

Por eza es logtro que of publico elen. se. como la misjorla de los jeles de luerias paplumentarias. que debers otorgar se el suplicitorio. Cnustiteremos que es el unico medio de pones pemate in asunto penosisimo y lamblén of tuzico de que sed igual la justicio para cuantas se vieron envuefios en al dessitre. Es paía ve sumsriados a muilios que en visperas del derrumbamienlo ejecutaran proczas merillsimas y a olros que, despuds, supleron vitudiearse en acelones dignas de elepio; cusndo lal oeurte, debe haber restmenes de ezception para nudie, absolusismente pata nadir.

Aozusto VIYEno.

EL ESTRENO DE "ELECTRA"

ise ocuertan ustedes de aguel dlodrid de hater veinte o velaticlace ofosl Lo que no lo recurerdea, llevan sobre noso. iros dos venlojas: uns lu novedad de es.

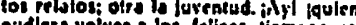
pudirra volvet a los telizes tiempos que callardeabsmos lat dizlochs prime

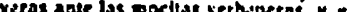
las sotrdes con possus y botitol Entonces le llamabsa a uno pollo y se enzallaba, hoz nos to liamas y has que dar un alori. Thquel Modris!

El couper-tanco, el thedenzante, L

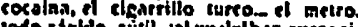
zoso ispleda, suitl, 101 ruclolihen expopsi2ado, has entanchada, has supersensi-

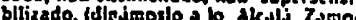
ra) ls copiligi de Espania: pero jall (otro

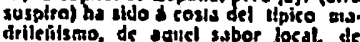
drilenitsmo, de agnel sabor locdt, de de cusntos nos adenardbamos en sy vi vir esslizo. Porgue diatrid es tan hospltatario, acepts las sentllarents lodos los gestins que en cusato yiss uno lu ca. ile de Sevilla por un miss, toma carla de mudriltrito neto. En Mlodrid, to que se dice en Aladtit . Tu ha naxido ninceuno de sus verinos, sepglin deria cons sucractio nalural Fellipe Lemesdex. Ni Durascal, il Antolitto Casero, apcear de to capa llereadd, ni el proplo Chleo de la Blusa que 80 ilpos represenlativos de la vi. tla del Oeo, har nacido en diadrid. Arsio nito Casero, seguta el mals berreas de Enrigur Gorrio Alverez, sesulie que rio la luz del sol ide la buas en Salamanea. $y$ de Vlecate Pastor cuents us pvisarso de Coblan que es de Guinzlo de Clmia johi al ladol

Pero voltiamos al dledrid de ctilonses. aquel que estaban en cervel de lo plas. de la carnetera de Arajón. diezzonlial, Fueriessilgatedo, Bombila iloretos machost En el Apolo. Mapolo Rodriguez Emitto Correras los Nlesefo y lodo e garbo y loda lo planerta de la Pino la Bru, la Pretel. En la politila brillaban

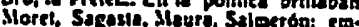
presben batellar, Lerrous Sorizao. Blasco listiez. Era ba tpore de Galdos. de Pereda de Eebeparay. del hisestro Cabaliero, de Chuece, de Chapl albo reaba, el esiro muske: de Quintro toi. verde. de Serrana el potilso de Ruben, de Vaile lactistro de Gabrisl y Galisa. Ers 1. tpoes en lin de acuiellas tertulias de calt, a las que thon kdo ecthando pose poco los ruitos del Janz-Banks.

Entonces los rstrenos en los leatros madriteños tenisa una solemenidad, Heraban ua sello de laisets i de pasion que ha desaparectido cati. [E gris es boy color de mods!

itn el saloncillo de Aprolo, una retarcis alarpsds y esirechi como colcads en un riarda del escenarto, cos reunie. mos duranic is leneiton cos Arrecui Arrue, aqueilla razon socisl que acredito el lesiro. alyunos de los primates del iriinestre iestrul de entonces, y olros de 


\section{Gaceta}
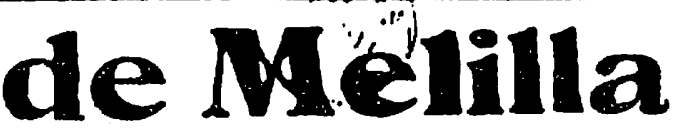

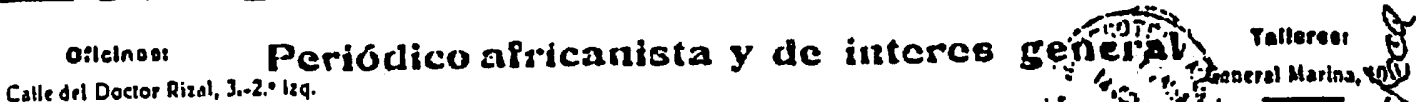
anunelos: Proclos uresa iollts

Diractor, Lundador u propletarlo. JAIntE TUR

\section{In Melilla se ha terminado el paro forzoso}

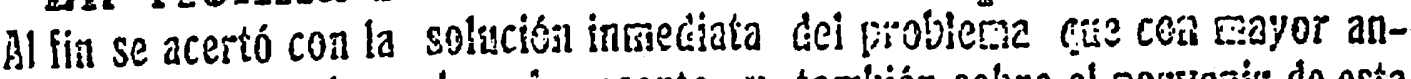
guitia se proyeciaba solbre el presente, y también sobre el gorvenit de esta ciudad, ansiosa de paz, de orden, de trabajo, y de procreso en tocios sias aspecios. Hubo sienpre las mejores intenciones, plasmocas en iniciativas muy laudables, pero carenties de basse suliciente, y de organizacién adecuada para

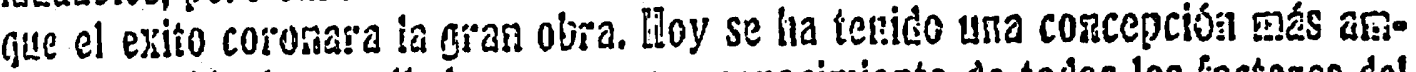
plia y ha sito desarroltada con mayn cortociniento de tolos los factores del gravisino conflicto. Es jasto reconecer el acierto del Delegado cel Eobierno,

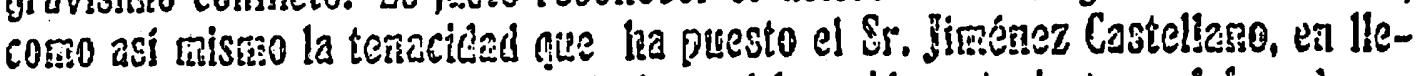
var adilante la magna emeresa, de la cual han sido cntusiostas colacioradores,

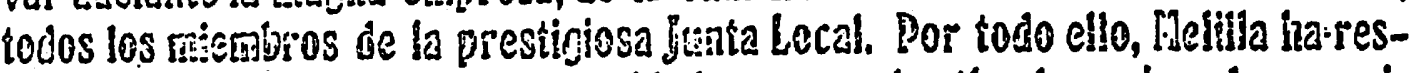
pondidio con sa tradicional generosidad, gue será estínalo y eicraplo a seguir por otras ciudades, amantes de sus obreros y trabajadores de todas clases. Jaime TUR

\section{La República es de to- dos y para todos}

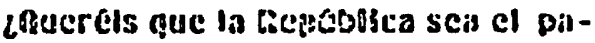
trimenlo de un partino? is cosis Qeirer age sen patrlosoblo de ua partisto al alre gue resplamos ca ta atmosfera $y$ la luz ac las extreltas. No; da Eccobltca es para lodos:to hepsifilca es per toces:

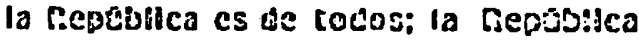
quedalado la Naclon tucerfona, es la Naclon misma,que recose su soberanta sotre todus sus biljor. como madre amorosa yue es de lodes cosotros."

\section{ERJILIO CASTELAR}

\section{La salud del pueblo}

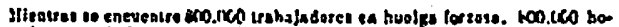

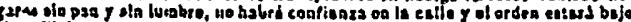

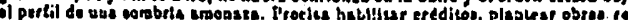

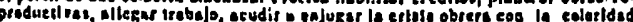

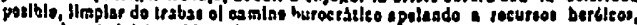

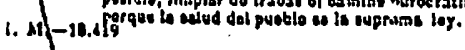

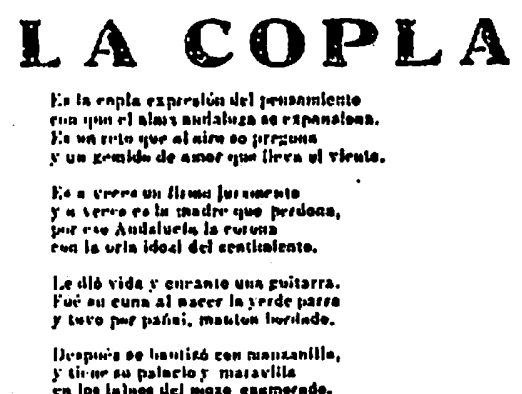

มลHЕدА มONT.SES

Ni trágalas ni coacciones

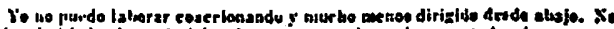

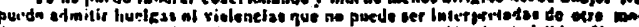

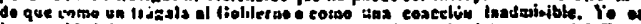

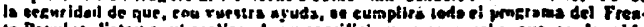

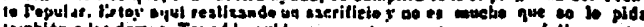

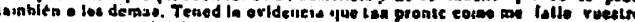

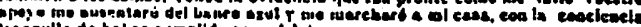
lisaqulls de helent eumpllde cen at deber.

HAXTIAOO CARARES YUUROGA

\section{Los Musulmanes y España}

FUEROM LOE LUKULAANES BUESTHOS YAESTROS, YLES URBEMDS

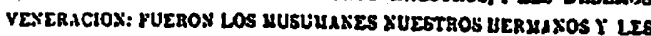
DERELOS ANOR; FUEHOX LOS MUSULACANES RUEST'PSá VICTIYAS Y LES DEREHOS REI'ARACJOX. 


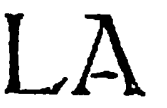

$:=: \because:===:-:==$ IULVISTA GRAIICA. LITERARIA Y DEPORTIVA Aino 1 -:- -:- Melilla 10 de Seplicinbre de 1928 -:- $\quad-$ Nim. 1 . -

E.I vilelo maraviltosn thial a Plus Llliran solure el

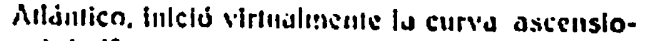
mal de lispana.

Quiches habfean siluadio a nutesira Pairla en el plibinis linlitilor de las Haclones decactentes, padecieron un crostsino error de persinecilva.

Cunfundicron Ins conceplos de Esiado y de $\mathrm{Na}$ ción, olviddudo. se de que las grandes virludes die una Raza creiluors, colo. inzaciors y clvilizadora de puseblos, podrán sufrlr ecllpses parclales, pero nuinca se ogolan, nI inuclio menos, inueren.

Pocosanosira mecesiliado Ispisfia.' mediante un goblerno fierie y estable, para demosirar al munto cline posee una vitalijad espiritilal y ecomumica, sorpre ufelle y eletno.

No sólo ha solido llegar a la cumbre de una prosperidad envidiable, sl no que, $y$ esto es mós diflell ain, ha logrado manlenterse ell ella, reallzimido empresiss dignas de sullistorla; ponlendo al mismo nivel sus gosios y sus ingresos, y acariclando el ldeal de una superacióa slempre. deseada, y liasta aliora, jamśs conseguida.

Eil la Llicralura, en el Arte, y en la Clencia imundiales, ocupa España un lugar preminen-

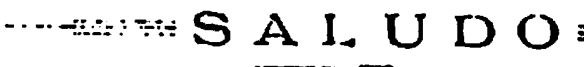

Silimus al noble palumpue de las Letras, perticechindos con min pubre bagajc: nitestra voluntad $y$ niestro entusiasmo. Pcro kinemos, cu cambio, un rico resoro de $f e ; f e$ ell mestros queididos camaradas; $f e$ en el público, cujo precioso apojo se nos brinda ja, 5. El ha de suplir vculajosamente a miestra incptilud r ha de inspirarnus el acicrlo para ser el portanoz, lal vef al mils mudesto y el mis humilute de muestra Espana ell cstas ticrras heroicas, pero finnco y leal, sin las reserinas mentules de los que desenumeluen su vida par el camino torthloso de las malas artes.

Sca, puce, nucstro saludo para las diguns autorillndes, para muestios colegras y para el pueblo melillense, pur el qui llegaremos haciu el sacrificio si cllo fucra ncicsario. le: y en la Mlillcia, lods la campaña marrogul. $y$ de modo muy espicial la epopejo de Alinucemas. dicen lo que nuestra liumilld piumo no - serla capaz de. expresar . debidameniz.

IY estamasen el morrento intclal de la ascen. sión!

No dudemos, por lo sonto,- de que con el noble $y$ entustasta esfuerzo de todos los elismenlos socic'es de nues tro pals, iveremos en el más hreve espaclo de liempo, a a nuesira gloriosa nactón, respetada por su gron poder, athimirada por sus exiraordinartas viriudes. $y$ su indiscuilble coniribución a la paz del mundo.

Jape TUR

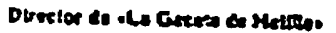




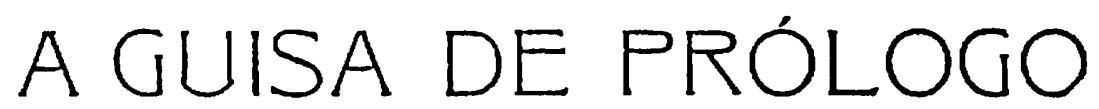

funotrs del ideal; heraldos de una raza que no muere, hemos querido
dejar a nuestro paso por estas tierras africanas algo perdurable y te las frescas brisas del mar azul....

España; la España de nuestros amores, al poner su planta en estas tiertas mogrebinas, no hace más que continuar la historia trazada en el año 711 por las huestes de Muza y de Taril. Aqui, conto alli, se impone la compenetración ideológica, de mucha más polencialidad que fuerza alguna ideada y de eleclo más positivo y lógico en el campo de la civilización.

Nuestra patria trae a estas tierras, tras la victoria de su heróico ejército los gérmenes de una era de progreso y de paz. El Estrecho, - como ha dicho muy bien un ilustre africanista - no es un rio que nos separa, sino un puente que nos acerca, y constanlemente, abrazados por los lazos de la Historia, el pueblo hispano-musulmán seguirn viviendo-tras pequeñas luchas fralricidasla era de esplendor y grandeza trazada por la mano del Destino.

He aqui los pasos a seguir por esta nuestra publicación. España y siempre España será nuestro lema de combate; y con el saludo fervoroso hacia nuestro Eiércilo y' los invictos caudillos que hoy le dirigen, y el alecto fraternal para los colegas hispano-africanos, hace VIDA MARroqu su modesta enIrada en el campo del periodismo, donde quiere vivir con dignidad y decoro, aportando su graníto de arena a la redentora obra de nuestra Patria.

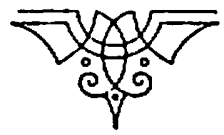

